TRANSACTIONS OF THE

AMERICAN MATHEMATICAL SOCIETY

Volume 361, Number 10, October 2009, Pages 5469-5504

S 0002-9947(09)04832-6

Article electronically published on April 21, 2009

\title{
TWISTING DERIVED EQUIVALENCES
}

\author{
OREN BEN-BASSAT
}

\begin{abstract}
We introduce a new method for "twisting" relative equivalences of derived categories of sheaves on two spaces over the same base. The first aspect of this is that the derived categories of sheaves on the spaces are twisted. They become derived categories of sheaves on gerbes living over spaces that are locally (on the base) isomorphic to the original spaces. Secondly, this is done in a compatible way so that the equivalence is maintained. We apply this method by proving the conjectures of Donagi and Pantev on dualities between gerbes on genus-one fibrations and comment on other applications to families of higher genus curves. We also include a related conjecture in Mirror Symmetry.
\end{abstract}

\section{INTRODUCTION}

Categories of sheaves of modules on geometric objects seem to play an important role in algebraic geometry. It is sometimes possible to analyze the algebraic properties of such categories in detail, which in turn sheds light on the nature of the space itself. On the other hand, they also suggest a broader perspective in which a space can vary in "non-geometric" directions, and eventually the notion of a space could be replaced by a category with certain properties. Gabriel showed in 21] that one can recover a Noetherian scheme from its category of coherent sheaves. Alternatively, one can study the derived category of coherent sheaves. This is a less rigid structure, and certainly allows for different spaces to have equivalent derived categories. This "derived equivalence" game started with Mukai's equivalence of dual complex tori [29, and we give a quick summary in what follows. The fundamental question which motivates this work (although we only scratch its surface) is, How does the derived category vary in families? Although the derived category is certainly known not to glue, in some cases it seems to behave as if it does. That is to say, if one is careful enough, one can prove things that would easily follow if descent for derived categories held. In this thesis, we investigate the special case when the gluing takes place on the level of the abelian category of sheaves (which as a stack can be glued from its restriction to a cover). Rather than directly study these questions for the derived category of a space, we study the variance (or twisting) of pairs of derived categories related by a Fourier-Mukai type equivalence. In practice, we start with a given Fourier-Mukai equivalence between the derived categories of two spaces $X$ and $Y$. We ask: If the category of sheaves on $X$ is twisted in some way, can the derived category of $Y$ be twisted in a compatible way in order to recover a new equivalence? On the infinitesimal level, this question was

Received by the editors December 14, 2007.

2000 Mathematics Subject Classification. Primary 14D22, 14K99, 18E30.

(C)2009 American Mathematical Society Reverts to public domain 28 years from publication 
addressed by Y. Toda in 32. A formal analysis to all orders was completed, in a special case, in 4. A complete picture of dualities for arbitrary gerby deformationquantizations of abelian schemes has been completed by D. Arinkin, building on the results from his 2002 thesis [3. A non-formal version of this same case was carried out by J. Block in [5, 6. Topological versions of twisted T-dualities have been studied by Mathai and Rosenberg in [27, 28, and by Bunke, Rumpf, and Schick in 12. It is somewhat interesting and strange that the philosophy of Toda from 32 will be helpful to us, even though our twistings are not necessarily deformations, and certainly not formal deformations. According to this philosophy, for every derived equivalence between $X$ and $Y$, one should be able to first find an algebraic object describing the twistings of the derived category $\mathrm{D}(X)$ compatible with the derived equivalence. Next one should find a natural isomorphism between these twistings of $\mathrm{D}(X)$ and the analogous twistings of $\mathrm{D}(Y)$. Finally, one should find an equivalence between the categories associated to a pair of compatible twistings. In this thesis, we synthesize two types of twistings. The first are those coming from replacing a space by another space locally isomorphic to it. The second are those coming from replacing the derived category of sheaves on a space with the derived category of sheaves on a gerbe over the space. The most convenient setting therefore becomes the one where $X$ and $Y$ are both fibered over the same space $B$, and the derived equivalence respects that structure. In this case, our twists of the derived categories correspond to decomposing and then re-gluing the relative stack of the abelian categories of sheaves of the two spaces. We can now state our main theorem (with slight re-wording), to be proven as Theorem 5.11. Here we use the definitions of $\Phi$-compatible and $\Phi$-dual, which can be found as Definitions 5.6 and 5.8 respectively.

Theorem 1.1. Let $X$ and $Y$ be compact, connected, complex manifolds, mapping to a complex analytic space $B$, via maps $\pi: X \rightarrow B$ and $\rho: Y \rightarrow B$, where $\rho$ is flat. Let $\mathcal{P}$ be a coherent sheaf, flat over $Y$, on the fiber product $X \times_{B} Y$, which gives an equivalence of categories $\Phi: D_{c}^{b}(Y) \rightarrow D_{c}^{b}(X), \Phi=R \phi$ with

$$
\phi(\mathcal{S})=\widetilde{\rho}_{*}\left(\mathcal{P} \otimes \tilde{\pi}^{*} \mathcal{S}\right) .
$$

Then for any $\Phi$-compatible gerbe $\mathfrak{X}$ over a twisted version of $X \rightarrow B$, and any $\Phi$-dual gerbe $\mathfrak{Y}$ to $\mathfrak{X}$, there is an equivalence of categories

$$
\widetilde{\Phi}: D_{c}^{b}(\mathfrak{Y},-1) \rightarrow D_{c}^{b}(\mathfrak{X},-1),
$$

where $\widetilde{\Phi}=R \widetilde{\phi}$ and $\widetilde{\phi}$ is locally built out of $\phi$.

The motivation for such a theorem begins at least with Dolgachev and Gross [18, who relate the Tate-Shafarevich group of an elliptic fibration to its Brauer group. This naturally leads one to ask if the derived category of a geometrically twisted elliptic fibration (a genus one fibration) is somehow related to a derived category of sheaves on a gerbe over the elliptic fibration. Such a relationship was indeed shown in [15, 14. More general dualities involving gerbes on genus one fibrations were proven in [19. The conjectures made in [19] form the main geometric motivation for this paper, although the methods of proof will be closer to those found in [14]. More recently, these kinds of results have been used and expanded upon by other authors; for example see [11, 13, and [31. An alternative, and in many ways stronger, version of the main theorem can be found as Theorem 5.12 . 
In the last section, we comment on some future, more exotic applications of the ideas we have developed, including applications to homological mirror symmetry and hyperelliptic families in algebraic geometry. This manuscript is based on the University of Pennsylvania thesis and some notes of the author which appeared as arXiv:math/0606631.

\section{Notation AND CONVENTIONS}

2.1. Basic information. In this paper, we work with complex manifolds or complex analytic spaces, and we always use the classical (analytic) topology. If $\mathcal{S}$ is a sheaf of abelian groups on a topological space $X$ and $\mathfrak{U}$ is a cover of $X$, then we denote by $\check{C}_{\mathfrak{U}}(X, \mathcal{S}), \check{Z}_{\mathfrak{U}}(X, \mathcal{S})$, and $\check{B}_{\mathfrak{U}}(X, \mathcal{S})$ the Cech co-chains, co-cycles, and co-boundaries for the sheaf of abelian groups $\mathcal{S}$ computed with respect to the cover $\mathfrak{U}$ of $X$. When we are dealing with a ringed space (or stack), by a sheaf (with no qualifications) we always mean a sheaf of modules for the structure sheaf of the space (or stack).

For our purposes, it will always suffice to consider stacks on a complex analytic space $X$ which are defined on the site whose underlying category consists of analytic spaces over $X$ and whose covering families for a given $W \rightarrow X$ consist of surjective local isomorphisms $\left\{W^{\prime} \rightarrow W\right\}$ over $X$. We will often consider the case $W=X$. For a map $W \rightarrow X$ we often use the convention

$$
\left.W^{k}=W \times_{X} W \times_{X} W \times_{X} \cdots \times_{X} W \quad \text { (the }(k+1) \text {-fold product }\right) .
$$

Since our methods are quite general, we expect that the results contained in this paper apply in other contexts such as the étale topology on schemes or for twisting different kinds of relative dualities in mathematics.

By a gerbe, we shall always mean a $\mathcal{O}^{\times}$-gerbe with trivial band on a complex analytic space. On a complex analytic space $X$, we use $\mathfrak{M o d}(X)$ to denote the stack of abelian categories of $\mathcal{O}_{X}$-modules, which has as global sections $\operatorname{Mod}(X)$. We use $\mathfrak{C o h}(X)(\mathfrak{Q} \mathfrak{C o h}(X))$ to denote the stack of abelian categories of coherent (quasi-coherent) $\mathcal{O}_{X}$-modules, which has as global sections the abelian category $\operatorname{Coh}(X)(\mathrm{QCoh}(X))$. We will also need to consider sheaves on gerbes $\mathfrak{X} \rightarrow X$. For every analytic space $Z$ mapping to $X$ by $f: Z \rightarrow X$, we consider the stack of functors $f^{-1} \mathfrak{X} \rightarrow f^{-1} \mathfrak{M o d}(X)$ and their natural transformations. This gives the stack of abelian categories of $\mathcal{O}_{\mathfrak{X}}$-modules $\mathfrak{M o d}(\mathfrak{X}) \rightarrow X$, and similarly we have the stack of abelian categories of coherent (quasi-coherent) $\mathcal{O}_{\mathfrak{X}}$-modules $\mathfrak{C o h}(\mathfrak{X}) \rightarrow X$ $(\mathfrak{Q} \mathfrak{C o h}(\mathfrak{X}) \rightarrow X)$, and we call their global sections the abelian categories $\operatorname{Mod}(\mathfrak{X})$ and $\operatorname{Coh}(\mathfrak{X})(\mathrm{QCoh}(\mathfrak{X}))$ respectively. We use $\mathrm{D}^{*}(X)$ and $\mathrm{D}^{*}(\mathfrak{X})$ to denote the derived categories of $\operatorname{Mod}(X)$ and $\operatorname{Mod}(\mathfrak{X})$. When nothing appears in the location of the $\operatorname{symbol} *$, or $*=\emptyset$, this refers to the unbounded derived categories. When $*=-$, this refers to the bounded above derived category, and when $*=b$, this refers to the bounded derived category. Also $\mathrm{D}_{c}^{*}(X)$ and $\mathrm{D}_{c}^{*}(\mathfrak{X})$ or $\mathrm{D}_{q c}^{*}(X)$ and $\mathrm{D}_{q c}^{*}(\mathfrak{X})$ refer to the derived categories of coherent and quasi-coherent sheaves. For a discussion of quasi-coherence in the complex analytic context, see [4]. The sheaves of weight $k$ on a gerbe will be denoted by $\mathfrak{M o d}(\mathfrak{X}, k), \mathfrak{C o h}(\mathfrak{X}, k), \mathfrak{Q} \mathfrak{C o h}(\mathfrak{X}, k)$ with global sections $\operatorname{Mod}(\mathfrak{X}, k), \operatorname{Coh}(\mathfrak{X}, k), \mathrm{QCoh}(\mathfrak{X}, k)$, with their associated derived categories $\mathrm{D}^{*}(\mathfrak{X}, k), \mathrm{D}_{c}^{*}(\mathfrak{X}, k)$, and $\mathrm{D}_{q c}^{*}(\mathfrak{X}, k)$. 


\section{BASIC TECHNIQUES}

3.1. Mukai's insight. In this section we review some well known facts, mainly due to Mukai [29]. None of the material in this section is original.

Given two complex analytic spaces $M$ and $N$ over $B$ and an element

$$
K \in \operatorname{Mod}\left(M \times_{B} N\right),
$$

the integral transform

$$
\phi_{K}^{[M \rightarrow N]}: \operatorname{Mod}(M) \rightarrow \operatorname{Mod}(N)
$$

is defined by

$$
\phi_{K}^{[M \rightarrow N]}(G)=p_{N *}\left(p_{M}^{*} G \otimes K\right) .
$$

Also for a map $f: M \rightarrow N$ over $B$, we have

$$
\phi_{\mathcal{O}_{\Gamma_{f}}}^{[M \rightarrow N]} \cong f_{\star}
$$

as functors $\operatorname{Mod}(M) \rightarrow \operatorname{Mod}(N)$ and

$$
\phi_{\mathcal{O}_{\Gamma_{f}}}^{[N \rightarrow M]} \cong f^{\star}
$$

as functors $\operatorname{Mod}(N) \rightarrow \operatorname{Mod}(M)$. For $L$ a line bundle on a space $M$, we use $T_{L}: \operatorname{Mod}(M) \rightarrow \operatorname{Mod}(M)$ to denote the functor

$$
\begin{array}{ll}
T_{L}(\mathcal{M}):=L \otimes \mathcal{M} & \forall \mathcal{M} \in \operatorname{ob}(\operatorname{Mod}(M)), \\
T_{L}(h):=\operatorname{id}_{L} \otimes h & \forall h \in \operatorname{Hom}_{M}\left(\mathcal{M}^{\prime}, \mathcal{M}^{\prime \prime}\right) .
\end{array}
$$

Then we have

$$
\phi_{\Delta_{*} L}^{[M \rightarrow M]} \cong T_{L},
$$

where $\Delta: M \rightarrow M \times_{B} M$ is the diagonal embedding. This isomorphism uses the symmetry property of the tensor product. Similarly, for $f$ an automorphism of $M$, and $L$ a line bundle on $M$, we can implement the functor $T_{L} \circ f^{*}$ by the object $(f, 1)_{*} L \in \operatorname{Mod}\left(M \times_{B} M\right)$. In other words, we have an isomorphism

$$
T_{L} \circ f^{*} \cong \phi_{(f, 1)_{*} L}^{[M \rightarrow M]} .
$$

This isomorphism uses the symmetry property of the tensor product.

Given two complex analytic spaces $M$ and $N$ and an element $\mathcal{K} \in \mathrm{D}\left(M \times{ }_{B} N\right)$ we denote by $\Phi_{K}^{[M \rightarrow N]}$ the functor

$$
\mathrm{D}(M) \rightarrow \mathrm{D}(N)
$$

given by

$$
\mathcal{S} \mapsto R \pi_{N *}\left(\mathcal{K} \otimes^{L} L \pi_{M}^{*} \mathcal{S}\right) .
$$

Now, as noted in [2], these transforms enjoy the following locality property. Fix flat maps $h: S \rightarrow B$ and $h^{\prime}: S^{\prime} \rightarrow S$. For us the most important case will be the inclusions of an open set. Let $M_{S}, N_{S}, M_{S^{\prime}}, N_{S^{\prime}}$ denote the fiber products. Let $\mathcal{K}_{S}$, and $K_{S^{\prime}}$ denote the derived pullbacks of $\mathcal{K}$ to $\mathrm{D}\left(M_{S} \times{ }_{S} N_{S}\right)$ and $\mathrm{D}\left(M_{S^{\prime}} \times{ }_{S^{\prime}} N_{S^{\prime}}\right)$ respectively. Let $h_{M}^{\prime}: M_{S^{\prime}} \rightarrow M_{S}$ and $h_{N}^{\prime}: N_{S^{\prime}} \rightarrow N_{S}$ be the canonical morphisms Then there is a natural isomorphism of functors

$$
L h_{N}^{\prime *} \circ \Phi_{\mathcal{K}_{S}} \cong \Phi_{\mathcal{K}_{S^{\prime}}} \circ L h_{M}^{*} .
$$


The integral transform has the following convolution property (see 29] or [30, Proposition 11.1]): If $M, N$ and $P$ are complex analytic spaces over $B$ and $\mathcal{K} \in$ $\mathrm{D}^{b}\left(M \times_{B} N\right)$ and $\mathcal{L} \in \mathrm{D}^{b}\left(N \times_{B} P\right)$, then one has a natural isomorphism of functors

$$
\Phi_{\mathcal{L}}^{[N \rightarrow P]} \circ \Phi_{\mathcal{K}}^{[M \rightarrow N]} \cong \Phi_{\mathcal{L} * \mathcal{K}}^{[M \rightarrow P]}
$$

where

$$
\mathcal{L} * \mathcal{K}=R p_{M \times_{B} P *}\left(L p_{N \times_{B} P}^{*} \mathcal{L} \otimes^{L} L p_{M \times_{B} N}^{*} \mathcal{K}\right) \quad \in \mathrm{D}^{b}\left(M \times_{B} P\right),
$$

and $p_{M \times N}, p_{M \times P}, p_{N \times P}$ are the natural projections $M \times_{B} N \times_{B} P \rightarrow M \times_{B} N$, etc.

This isomorphism of functors is compatible with base change, in the sense that if we consider the maps

$$
\begin{gathered}
h_{M N}: M_{S} \times_{S} N_{S} \rightarrow M \times_{B} N, \\
h_{N P}: N_{S} \times{ }_{S} P_{S} \rightarrow N \times{ }_{B} P,
\end{gathered}
$$

and

we have

$$
h_{M P}: M_{S} \times_{S} P_{S} \rightarrow M \times_{B} P,
$$

$$
\left(L h_{N P}^{*} \mathcal{L}\right) \star\left(L h_{M N}^{*} \mathcal{K}\right) \cong L h_{M P}^{*}(\mathcal{L} \star \mathcal{K}) \in \mathrm{D}\left(M_{S} \times{ }_{S} P_{S}\right)
$$

Also, from Huybrechts' book [23, we have that

- for any $f: N \rightarrow P$ over $B$ we have the natural isomorphism of functors $\mathrm{D}^{b}(M) \rightarrow \mathrm{D}^{b}(P)$

$$
f_{*} \circ \Phi_{K}^{[M \rightarrow N]} \cong \Phi_{\left(1_{M} \times f\right)_{*} K}^{[M \rightarrow P]},
$$

- for $f: P \rightarrow N$ over $B$ we have the natural isomorphism of functors $\mathrm{D}^{b}(M) \rightarrow \mathrm{D}^{b}(P)$

$$
f^{*} \circ \Phi_{K}^{[M \rightarrow N]} \cong \Phi_{\left(1_{M} \times f\right)^{*} K}^{[M \rightarrow P]},
$$

- for $g: W \rightarrow M$ over $B$ we have the natural isomorphism of functors $\mathrm{D}^{b}(M) \rightarrow \mathrm{D}^{b}(P)$

$$
\Phi_{K}^{[M \rightarrow N]} \circ g_{*} \cong \Phi_{(g \times 1)^{*} K}^{[W \rightarrow N]},
$$

- for $g: M \rightarrow W$ over $B$ we have the natural isomorphism of functors $\mathrm{D}^{b}(M) \rightarrow \mathrm{D}^{b}(P)$

$$
\Phi_{K}^{[M \rightarrow N]} \circ g^{*} \cong \Phi_{(g \times 1)_{*} K}^{[W \rightarrow N]} .
$$

Of course we also have the analogue of equation (3.1) in the derived context, which is an isomorphism of functors $\mathrm{D}^{b}(M) \rightarrow \mathrm{D}^{b}(M)$, for any automorphism $f$ of $M$, and line bundle $L$ on $M$ :

$$
T_{L} \circ f^{*} \cong \Phi_{(f, 1)_{*} L}^{[M \rightarrow M]}
$$

For a gerbe $\mathfrak{X}$, it is a generally known fact that the category $\operatorname{Mod}(\mathfrak{X})$ has enough injective and enough flat objects; see for example [14] and [26]. Indeed, following [26], if $\mathfrak{a}: \mathcal{U} \rightarrow \mathfrak{X}$ is an atlas and $\mathcal{M}$ is a sheaf on $\mathfrak{X}$, then we can find an injection

$$
\mathfrak{a}^{*} \mathcal{M} \rightarrow \mathcal{I}
$$

where $\mathcal{I}$ is an injective $\mathcal{O}_{\mathcal{U}}$-module. We can also find a surjection

$$
\mathcal{F} \rightarrow \mathfrak{a}^{*} \mathcal{M},
$$


where $\mathcal{F}$ is a flat $\mathcal{O}_{\mathcal{U}}$-module. Therefore, we have a sequence of injections

$$
\mathcal{M} \rightarrow \mathfrak{a}_{*} \mathfrak{a}^{*} \mathcal{M} \rightarrow \mathfrak{a}_{*} \mathcal{I}
$$

where $\mathfrak{a}_{*} \mathcal{I}$ is injective, and a sequence of surjections

$$
\mathfrak{a} ! \mathcal{F} \rightarrow \mathfrak{a} ! \mathfrak{a}^{*} \mathcal{M} \rightarrow \mathcal{M}
$$

where $\mathfrak{a}_{!} \mathcal{F}$ is flat.

3.2. Some functors. Recall that for $X$ and $Y$ complex analytic spaces, a gerbe $\mathfrak{Y} \rightarrow Y$ and a morphism $f: X \rightarrow Y$, we can form the $f^{-1} \mathcal{O}_{Y}^{\times}$banded gerbe $f^{-1} \mathfrak{Y}$, and then via the map of sheaves of groups $f^{-1} \mathcal{O}_{Y}^{\times} \rightarrow \mathcal{O}_{X}^{\times}$, we define an $\left(\mathcal{O}_{X}^{\times}\right.$-banded) gerbe $f^{*} \mathfrak{Y}$ on $X$.

We will often use the left exact functor $f_{*}$, and the right exact functor $f^{*}$ :

- $f_{*}: \operatorname{Mod}\left(f^{*} \mathfrak{Y}\right) \rightarrow \operatorname{Mod}(\mathfrak{Y})$,

- $f^{*}: \operatorname{Mod}(\mathfrak{Y}) \rightarrow \operatorname{Mod}\left(f^{*} \mathfrak{Y}\right)$.

The first of these is defined by the composition

$$
\mathfrak{Y} \rightarrow f_{*} f^{*} \mathfrak{Y} \rightarrow f_{*} \mathfrak{M o d}(X) \rightarrow \mathfrak{M o d}(Y)
$$

The second is defined by the composition

$$
f^{*} \mathfrak{Y} \rightarrow f^{*} \mathfrak{M o d}(Y) \rightarrow \mathfrak{M o d}(X) .
$$

Also if we have two gerbes $\mathfrak{X} \rightarrow X$ and $\mathfrak{Y} \rightarrow X$ over the same space, we can define the gerbe $\mathfrak{X} \otimes \mathfrak{Y} \rightarrow X$ to be the $\mathcal{O}_{X}^{\times}$gerbe induced from the $\mathcal{O}_{X}^{\times} \times \mathcal{O}_{X}^{\times}$gerbe $\mathfrak{X} \times \mathfrak{Y}$ via the map of sheaves of groups $\mathcal{O}_{X}^{\times} \times \mathcal{O}_{X}^{\times} \rightarrow \mathcal{O}_{X}^{\times},(a, b) \mapsto a b$.

If we are given $\mathcal{M} \in \operatorname{ob}(\operatorname{Mod}(\mathfrak{X}))$ and $\mathcal{N} \in \operatorname{ob}(\operatorname{Mod}(\mathfrak{Y}))$, then we can form

$$
\mathcal{M} \otimes \mathcal{N} \in \operatorname{ob}(\operatorname{Mod}(\mathfrak{X} \otimes \mathfrak{Y})) .
$$

This leads, for $\mathcal{N} \in \operatorname{ob}(\operatorname{Mod}(\mathfrak{X}))$, to the right exact functor $T_{\mathcal{N}}$

$$
T_{\mathcal{N}}: \operatorname{Mod}(\mathfrak{X}) \rightarrow \operatorname{Mod}(\mathfrak{X} \otimes \mathfrak{Y}) .
$$

This is given by the composition

$$
\mathfrak{X} \otimes \mathfrak{Y} \rightarrow \mathfrak{X} \otimes \mathfrak{M o d}(X) \rightarrow \mathfrak{M o d}(X) \otimes \mathfrak{M o d}(X) \rightarrow \mathfrak{M o d}(X) .
$$

3.3. Some derived functors. A morphism $f: X \rightarrow Y$ is flat if the stalk $\mathcal{O}_{X, x}$ is a flat $\mathcal{O}_{Y, f(x)}$ module for all points $x \in X$. The flatness of $f$ is equivalent to the exactness of $f^{*}$. If $\mathcal{F}$ is a sheaf on $X$, then $\mathcal{F}$ is said to be flat over $Y$ if $\mathcal{F}_{x}$ is a flat $\mathcal{O}_{Y, f(x)}$ module for all points $x \in X$. If $\mathcal{F}$ is flat over $Y$ and $f$ is flat, then the functor $T_{\mathcal{F}} \circ f^{*}$ is exact.

If $f$ is flat, we have the derived functor

$$
f^{*}: \mathrm{D}(\mathfrak{Y}) \rightarrow \mathrm{D}\left(f^{*} \mathfrak{Y}\right),
$$

obtained by applying $f^{*}$ term by term to complexes. This functor obviously preserves the three types of boundedness. For a general map $f$ we have the derived functors

$$
\begin{aligned}
& R f_{*}: \mathrm{D}^{+}\left(f^{*} \mathfrak{Y}\right) \rightarrow \mathrm{D}^{+}(\mathfrak{Y}), \\
& L f^{*}: \mathrm{D}^{-}(\mathfrak{Y}) \rightarrow \mathrm{D}^{-}\left(f^{*} \mathfrak{Y}\right),
\end{aligned}
$$

and

$$
L T_{\mathcal{N}}: \mathrm{D}^{-}(\mathfrak{X}) \rightarrow \mathrm{D}^{-}(\mathfrak{X} \otimes \mathfrak{Y}) .
$$


3.4. Presentations of gerbes. Let $W$ be an analytic space. A presentation of a gerbe on $W$ is defined to be the following:

- an analytic space $\mathcal{U}$ mapping to $W$ by a surjective local isomorphism (atlas) to $W$ :

$$
a: \mathcal{U} \rightarrow W
$$

- a line bundle $L$ over $\mathcal{U} \times_{W} \mathcal{U}$; we will denote the left and right $\mathcal{O}$-module structures on $L$ by

$$
l: \mathcal{O} \otimes_{\mathbb{C}} L \rightarrow L \quad \text { and } \quad r: L \otimes_{\mathbb{C}} \mathcal{O} \rightarrow L,
$$

- an isomorphism

over $\mathcal{U} \times_{W} \mathcal{U} \times_{W} \mathcal{U}$

$$
\theta: p_{12}^{*} L \otimes p_{01}^{*} L \rightarrow p_{02}^{*} L
$$

- an isomorphism

$$
\eta: \mathcal{O} \rightarrow \Delta^{*} L
$$

over $\mathcal{U}$, where $\Delta: \mathcal{U} \rightarrow \mathcal{U} \times_{W} \mathcal{U}$ is the diagonal map

satisfying the conditions

- that there is an equality of isomorphisms

$$
p_{013}^{*} \theta \circ\left(p_{123}^{*} \theta \times 1_{p_{01}^{*} L}\right)=p_{023}^{*} \theta \circ\left(1_{p_{23}^{*} L} \times p_{012}^{*} \theta\right),
$$

$$
\begin{aligned}
& \left(1_{\mathcal{U}} \times \Delta\right)^{*} \theta \circ\left(p_{1}^{*} \eta \times 1_{L}\right)=r, \\
& \left(\Delta \times 1_{\mathcal{U}}\right)^{*} \theta \circ\left(1_{L} \times p_{0}^{*} \eta\right)=l .
\end{aligned}
$$

We will denote the presentation of a gerbe over $W$ defined in this way by

$$
(L, \theta, \eta) W \text {. }
$$

Here, we suppress the information of the map $a: \mathcal{U} \rightarrow W$ in the notation, assuming that this map is clear from the context.

Definition 3.1. A strong equivalence of two presentations of gerbes with atlas $a: \mathcal{U} \rightarrow W_{(L, \theta, \eta)} W$ and ${ }_{\left(L^{\prime}, \theta^{\prime}, \eta^{\prime}\right)} W$ is an isomorphism $\mu: L \rightarrow L^{\prime}$ that satisfies

$$
p_{02}^{*} \mu \circ \theta=\theta^{\prime} \circ\left(\left(p_{12}^{*} \mu\right) \otimes\left(p_{01}^{*} \mu\right)\right)
$$

and

$$
\delta^{*} \mu \circ \eta=\eta^{\prime}
$$

For completeness we note that a weak equivalence of two presentations of gerbes $(L, \theta, \eta) W$ and ${ }_{\left(L^{\prime}, \theta^{\prime}, \eta^{\prime}\right)} W$ over $a: \mathcal{U} \rightarrow W$ is a pair $(Q, \tau)$ consisting of

- a line bundle $Q \rightarrow \mathcal{U}$,

- an isomorphism

$$
\tau: L \otimes p_{0}^{*} Q \rightarrow p_{1}^{*} Q \otimes L^{\prime},
$$

satisfying the equality of isomorphisms

$$
p_{12}^{*} L \otimes p_{01}^{*} L \otimes p_{0}^{*} Q \rightarrow p_{2}^{*} Q \otimes p_{02}^{*} L^{\prime}
$$

given by

$$
p_{02}^{*} \tau \circ\left(\theta \otimes 1_{p_{0}^{*} Q}\right)=\left(1_{p_{2}^{*} Q} \otimes \theta^{\prime}\right) \circ\left(p_{12}^{*} \tau \otimes 1_{p_{01}^{*} L^{\prime}}\right) \circ\left(1_{p_{12}^{*} L} \otimes p_{01}^{*} \tau\right)
$$

and the equality of isomorphisms

$$
Q \otimes \Delta^{*} L \rightarrow Q
$$


given by

$$
r \circ\left(1_{Q} \otimes \eta\right)^{-1}=l \circ\left(\eta^{\prime} \otimes 1_{Q}\right)^{-1} \circ \Delta^{*} \tau,
$$

where we have inserted the natural isomorphisms

$$
Q \otimes \Delta^{*} L \cong \Delta^{*}\left(p_{1}^{*} Q \otimes L\right) \quad \text { and } \quad \Delta^{*} L^{\prime} \otimes M \cong \Delta^{*}\left(L^{\prime} \otimes p_{0}^{*} Q\right) .
$$

3.5. Sheaves on presentations of gerbes. A sheaf of weight $k$ on a presentation of a gerbe with atlas $\mathcal{U} \rightarrow W$ is

- a sheaf $\mathcal{S}$ of $\mathcal{O}_{\mathcal{U}}$-modules over $\mathcal{U}$,

- an isomorphism $p_{0}^{*} \mathcal{S} \rightarrow\left(p_{1}^{*} \mathcal{S}\right) \otimes L^{k}$,

- which satisfies the extra condition that the following diagram commutes:

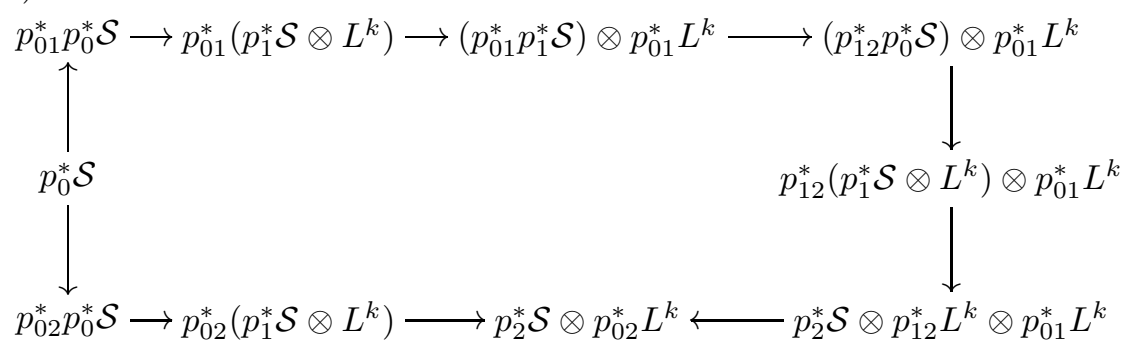

The sheaves on a presentation of a gerbe form an abelian category in the obvious way; a morphism between such sheaves $\mathcal{S}$ and $\mathcal{T}$ is simply a map $\mathcal{S} \rightarrow \mathcal{T}$ of sheaves on $\mathcal{U}$ such that the diagram below commutes:

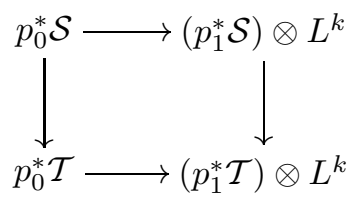

In this way we get abelian categories $\operatorname{Mod}\left({ }_{(L, \theta, \eta)} W, k\right)$ and $\operatorname{Coh}\left({ }_{(L, \theta, \eta)} W, k\right)$. They can also be seen as the categories of cartesian sheaves on the appropriate semisimplicial space.

For any presentation ${ }_{(L, \theta, \eta)} W$ of a gerbe $\mathfrak{W} \rightarrow W$, we have isomorphisms

$$
\operatorname{Mod}\left({ }_{(L, \theta, \eta)} W, k\right) \rightarrow \operatorname{Mod}(\mathfrak{W}, k)
$$

and

$$
\operatorname{Coh}\left({ }_{(L, \theta, \eta)} W, k\right) \rightarrow \operatorname{Coh}(\mathfrak{W}, k) .
$$

Remark 3.2. Two presentations of gerbes on the same cover that are strongly equivalent as defined in Definition 3.1 have equivalent categories of sheaves.

3.6. Derived pushforward, pullback, and tensor product. As mentioned before, there are some abstract ways to see that the category of sheaves on a gerbe possess injective and flat objects. To improve this slightly, we would like to see that there are flat and injective objects in $\operatorname{Mod}\left({ }_{(L, \theta, \eta)} W\right) \cong \operatorname{Mod}(\mathfrak{W})$ which map to the same type of objects in $\operatorname{Mod}(\mathcal{U})$. For this purpose consider the commutative 
diagrams

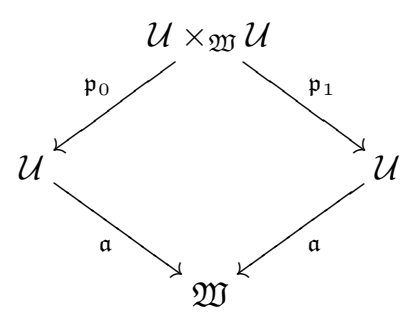

and

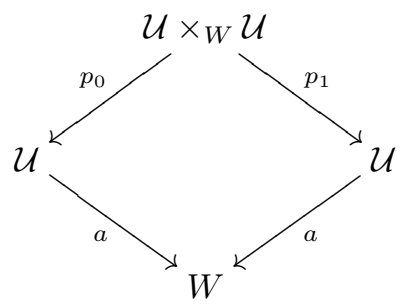

Notice that for any sheaf $\mathcal{M}$ of weight $k$ on $\mathfrak{W}$ equation (3.8) gives the existence of an injection $\mathfrak{a}^{*} \mathcal{M} \rightarrow \mathfrak{a}^{*} \mathfrak{a}_{*} \mathcal{I}$ for an injective sheaf $\mathcal{I}$ on $\mathcal{U}$. Flat base change then implies the existence of an injection in $\operatorname{Mod}\left({ }_{(L, \theta, \eta)} W\right)$ :

$$
\mathfrak{a}^{*} \mathcal{M} \rightarrow \mathfrak{a}^{*} \mathfrak{a}_{*} \mathcal{I} \cong \mathfrak{p}_{0 *} \mathfrak{p}_{1}^{*} \mathcal{I} \cong p_{0 *}\left(L^{k} \otimes p_{1}^{*} \mathcal{I}\right)
$$

Furthermore, $p_{0 *}\left(L^{k} \otimes p_{1}^{*} \mathcal{I}\right)$ is an injective sheaf on $\mathcal{U}$. Indeed since $p_{1}$ is a local isomorphism, it is clear that $p_{1}^{*} \mathcal{I}$ is a locally injective sheaf. Then, by 25. Proposition 2.4.10], we conclude that $p_{1}^{*} \mathcal{I}$ is injective. For any sheaf $\mathcal{N}$, we have $\operatorname{Hom}\left(\mathcal{N}, L^{k} \otimes p_{1}^{*} \mathcal{I}\right) \cong \operatorname{Hom}\left(\mathcal{N} \otimes L^{-k}, p_{1}^{*} \mathcal{I}\right)$, and tensoring with $L^{-k}$ is exact, so

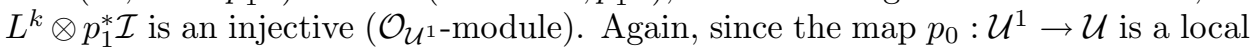
isomorphism we have that $\mathcal{O}_{\mathcal{U}^{1}}=p_{0}^{-1} \mathcal{O}_{\mathcal{U}}$. Finally, by [25, Proposition 2.4.1 (ii)] we have that $p_{0 *}\left(L^{k} \otimes p_{1}^{*} \mathcal{I}\right)$ is injective.

Similarly, equation (3.9) gives a surjection $\mathfrak{a}^{*} \mathfrak{a} !$ on $\mathcal{U}$. Flat base change gives a surjection in $\operatorname{Mod}\left({ }_{(L, \theta, \eta)} W\right)$ :

$$
p_{0 !}\left(L^{k} \otimes p_{1}^{*} \mathcal{F}\right) \cong \mathfrak{p}_{0 !}\left(\mathfrak{p}_{1}^{*} \mathcal{F}\right) \cong \mathfrak{a}^{*} \mathfrak{a}_{!} \mathcal{F} \rightarrow \mathfrak{a}^{*} \mathcal{M}
$$

Now since $p_{1}$ is a local isomorphism, $p_{1}^{*} \mathcal{F}$ is flat. Then it is clear that $L^{k} \otimes p_{1}^{*} \mathcal{F}$ is also flat, and flatness is preserved by $p_{0 !}$, so we conclude that $p_{0 !}\left(L^{k} \otimes p_{1}^{*} \mathcal{F}\right)$ is flat.

Suppose now that we have a commutative diagram

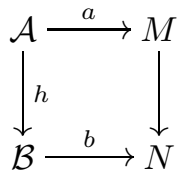

where $\mathcal{A}$ is an atlas for $M, \mathcal{B}$ is an atlas for $N$, and that the map $h$ induces a map $\tilde{h}: M \rightarrow N$. Furthermore, suppose we are given a gerbe $\mathfrak{N}$ on $N$. Chose a gerbe presentation $_{(L, \theta, \eta)} N$ of $\mathfrak{N}$ using $\mathcal{B}$ and also consider the pullback presentation $\left(h^{*} L, h^{*} \theta, h^{*} \eta\right) N$ of $h^{*} \mathfrak{N}$ on the atlas $\mathcal{A}$. Then using resolutions as we have described above (simultaneously flat or injective on both the atlas and on the presentation), 
we see that the following diagrams commute:

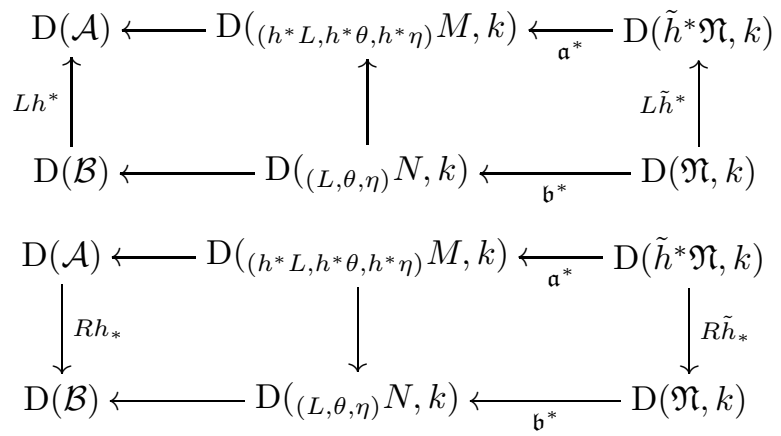

Also for any object $\mathcal{S}$ of $\mathrm{D}(\mathfrak{N}, c)$ and its associated object $\mathfrak{a}^{*} \mathcal{S}$ of $\mathrm{D}\left({ }_{(L, \theta, \eta)} N, c\right)$, we have the following commutative diagram:

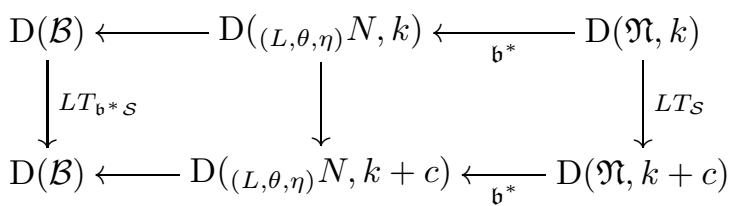

Notice that when thinking in terms of this presentation, we have for every $k \in \mathbb{Z}$ an adjoint pair of functors $\left(\mathfrak{a}_{k}^{*}, \mathfrak{a}_{k *}\right)$,

$$
\begin{gathered}
\mathfrak{a}_{k}^{*}: \operatorname{Mod}\left({ }_{(L, \theta, \eta)} W, k\right) \rightarrow \operatorname{Mod}(\mathcal{U}), \\
\mathfrak{a}_{k, *}: \operatorname{Mod}(\mathcal{U}) \rightarrow \operatorname{Mod}\left({ }_{(L, \theta, \eta)} W, k\right) .
\end{gathered}
$$

Here $\mathfrak{a}_{k *}(\mathcal{S})=p_{0 *}\left(L^{k} \otimes p_{1}^{*} \mathcal{S}\right)$. We will later use this in the form

$$
\mathfrak{a}_{-k *}(\mathcal{S})=p_{1 *}\left(L^{k} \otimes p_{0}^{*} \mathcal{S}\right),
$$

when we will be interested in the case $k=1$. Also note that $\mathfrak{a}_{k}^{*}$ is the obvious forgetful map.

\section{Constructing NeW Presentations from old}

Let $\pi: X \rightarrow B$ be any map, $\mathcal{B}=\left\{U_{i}\right\}$ an open cover of $B$, with atlas

$$
U=\coprod_{i} U_{i} \rightarrow B
$$

and $\mathcal{U}$ the pullback of this open cover by $\pi$. In other words we will use the atlas

$$
a: \mathcal{U}=\coprod_{i} \pi^{-1}\left(U_{i}\right) \rightarrow X
$$

We will consider the space $X$ to be presented by the diagram

$$
\mathcal{U} \times{ }_{X} \mathcal{U} \stackrel{p_{0}^{1}}{\stackrel{p_{1}^{1}}{\longrightarrow}} \mathcal{U},
$$

where the two maps are the obvious projections, or in other words,

$$
\coprod_{i j} \pi^{-1}\left(U_{i j}\right) \stackrel{p_{0}^{1}}{\underset{p_{1}^{1}}{\longrightarrow}} \coprod_{i} \pi^{-1}\left(U_{i}\right),
$$


where $p_{0}^{1}: \pi^{-1}\left(U_{i j}\right) \rightarrow \pi^{-1}\left(U_{i}\right)$ and $p_{1}^{1}: \pi^{-1}\left(U_{i j}\right) \rightarrow \pi^{-1}\left(U_{j}\right)$ become the obvious inclusion maps. Let $\Delta: \mathcal{U} \rightarrow \mathcal{U} \times_{X} \mathcal{U}$ be the diagonal map. This groupoid gives rise to a simplicial space $\mathcal{U}^{\bullet}$ resolving $X$ over $B$ of the form

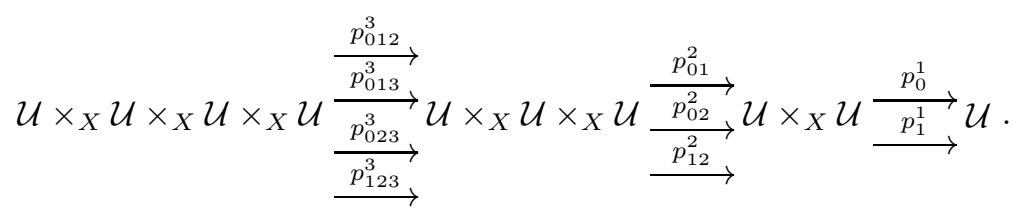

Here we use the maps

$$
p_{i_{0}, \ldots, i_{n}}^{n}: \mathcal{U}^{n+1} \rightarrow \mathcal{U}^{n}
$$

given by

$$
p_{i_{0}, \ldots, i_{n-1}}^{n}\left(u_{0}, \ldots, u_{n}\right)=\left(u_{i_{0}}, \ldots, u_{i_{n-1}}\right) .
$$

We will not need the degeneracy maps too much, but they are the obvious maps induced by the diagonal. We will consider the sheaf of groups $A u t(X / B)$ on $B$. A section of this sheaf over an open set $U \subset B$ is just an automorphism of $\pi^{-1}(U)$ which commutes with the projection to the base. To every element $f$ of $\check{Z}_{\mathfrak{B}}^{1}(B, A u t(X / B))$ we will associate a new analytic space $\pi_{f}: X_{f} \rightarrow B$.

Let $\pi^{n}: \mathcal{U}^{n} \rightarrow B$ be the obvious projection maps and consider the automorphism $f$ of $\mathcal{U}^{1}=\mathcal{U} \times{ }_{X} \mathcal{U}$ satisfying $\pi^{1}=\pi^{1} \circ f$. We will denote the restriction of $f$ to the component $\pi^{-1}\left(U_{i j}\right)$ by $f_{i j}$. On the space $\mathcal{U}^{a}$, we write $f_{c d}^{a}: \mathcal{U}^{a} \rightarrow \mathcal{U}^{a}$ for the restriction of $f$ to $\mathcal{U}^{a}$ via the map

$$
p_{c d}^{a}: \mathcal{U}^{a} \rightarrow \mathcal{U} \times{ }_{X} \mathcal{U}
$$

Therefore we have the equality $p_{c d}^{a} \circ f_{c d}^{a}=f \circ p_{c d}^{a}$, and of course $f=f_{01}$. The fact that $f$ is in $\check{Z}^{1}(B, \mathcal{B}, A u t(X / B))$ just means that $f_{01}^{2} \circ f_{12}^{2}=f_{02}^{2}$ and that $f \circ \Delta=\Delta$. We will often just write this more conveniently as $f_{i j} \circ f_{j k}=f_{i k}$ on $\pi^{-1}\left(U_{i j k}\right)$ and as $f_{i i}=1$ on $\pi^{-1}\left(U_{i i}\right)=\pi^{-1}\left(U_{i}\right)$.

The map $f$ defines a new analytic space $X_{f}$, and the map $\pi_{f}: X_{f} \rightarrow B$ is now defined by the presentation

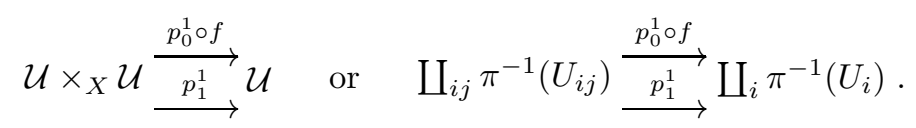

In other words, $X_{f}=\mathcal{U} / \sim$, where we identify a point $v \in \pi^{-1}\left(U_{j}\right)$ with $f_{i j}(v) \in$ $\pi^{-1}\left(U_{i}\right)$ as long as these points live over $U_{i j}$.

Notice that

$$
\coprod_{i j} \pi^{-1}\left(U_{i j}\right) \stackrel{p_{0}^{1} \circ f}{\stackrel{p_{1}^{1}}{\longrightarrow}} \coprod_{i} \pi^{-1}\left(U_{i}\right)
$$

is isomorphic to the diagram

$$
\coprod_{i j} \pi_{f}^{-1}\left(U_{i j}\right) \longrightarrow \coprod_{i} \pi_{f}^{-1}\left(U_{i}\right)
$$

(where we take the standard inclusions for the top and bottom arrows) via the isomorphisms

$$
\pi^{-1}\left(U_{i j}\right) \cong U_{i} \times_{B} \pi^{-1}\left(U_{j}\right)=U_{i} \times_{B} \pi_{f}^{-1}\left(U_{j}\right) \cong \pi_{f}^{-1}\left(U_{i j}\right) .
$$


This is perhaps more transparent in the other notation where we have the commutative diagram

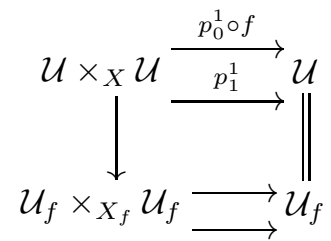

where the vertical arrow on the left hand side is the map $\left(p_{0}^{1} \circ f, p_{1}^{1}\right)$. In what follows, we will usually use the top row as a presentation of $X_{f}$. Of course, an equivalence of two presentations of the twisted fibrations $X_{f}$ and $X_{f^{\prime}}$, given by $f$ and $f^{\prime}$, is given by an automorphism $h$ of $\mathcal{U}$ which satisfies $\pi \circ h=\pi$, and if we let $h_{c}$ denote the restriction of $h$ to $\mathcal{U} \times_{X} \mathcal{U}$, via $p_{c}: \mathcal{U} \times_{X} \mathcal{U} \rightarrow \mathcal{U}$,

$$
h_{0} \circ f=f^{\prime} \circ h_{1} .
$$

This will often be written as $h_{i} \circ f_{i j}=f_{i j}^{\prime} \circ h_{j}$ on the component $\pi^{-1}\left(U_{i j}\right)$, where $h_{i}$ is just $h$ on the component $\pi^{-1}\left(U_{i}\right)$.

Define

$$
m_{j}^{n}:\left(\mathcal{U}^{n}\right)_{f} \rightarrow\left(\mathcal{U}^{n-1}\right)_{f}
$$

by

$$
m_{0, \ldots, n-1}^{n}=p_{0, \ldots, n-1}^{n} \circ f_{n-1, n}
$$

and for any $j$ with $0 \leq j \leq n-1\left(i_{0}, \ldots, i_{n-1}\right)$ with $0 \leq i_{0}<i_{1}<\cdots<i_{n-1} \leq n$, and $\left(i_{0}, \ldots, i_{n-1}\right) \neq(0, \ldots, n-1)$ let

$$
m_{i_{0}, \ldots, i_{n-1}}^{n}=p_{i_{0}, \ldots, i_{n-1}}^{n} .
$$

This defines a new simplicial manifold $\left(\mathcal{U}^{\bullet}\right)_{f}$ whose first terms look like

$$
\mathcal{U} \times_{X} \mathcal{U} \times_{X} \mathcal{U} \times_{X} \mathcal{U} \underset{\frac{p_{012}^{3} \circ f_{23}}{\stackrel{p_{013}^{3}}{\longrightarrow}}}{\stackrel{p_{023}^{3}}{\longrightarrow}} \mathcal{U} \times_{X} \mathcal{U} \times_{X} \mathcal{U} \underset{p_{123}^{3}}{\stackrel{p_{012}^{2} \circ f_{12}}{\longrightarrow}} \mathcal{U} \times \times_{X} \mathcal{U} \stackrel{p_{0}^{1} \circ f}{\longrightarrow} \mathcal{U}
$$

and the degeneracy maps are induced by $f$ and the diagonal. We have an isomorphism of simplicial manifolds

$$
\left(\mathcal{U}^{\bullet}\right)_{f} \cong\left(\mathcal{U}_{f}\right)^{\bullet}
$$

Its $n$-th component

$$
\left(\mathcal{U}^{n}\right)_{f} \rightarrow\left(\mathcal{U}_{f}\right)^{n}
$$

is given by

$$
\begin{aligned}
\pi^{-1}\left(U_{t_{0}, t_{1}, \ldots, t_{n}}\right) & \cong U_{t_{0}, \ldots, t_{n-1}} \times_{B} \pi^{-1}\left(U_{t_{n}}\right)=U_{t_{0}, \ldots, t_{n-1}} \times_{B} \pi_{f}^{-1}\left(U_{t_{n}}\right) \\
& \cong \pi_{f}^{-1}\left(U_{t_{0}, t_{1}, \ldots, t_{n}}\right) .
\end{aligned}
$$


4.1. Presentations of gerbes on twisted fibrations. In order to define presentations of gerbes on the space $X_{f}$, we will need to consider the terms

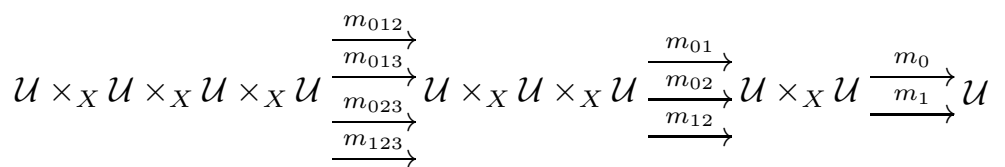

coming from the resolution of $X_{f}$ over $B$ described above. Due to the above remark, we see that a presentation of an $\mathcal{O}^{\times}$gerbe on $X_{f}$ is defined by a line bundle $L$ on $\mathcal{U} \times{ }_{X} \mathcal{U}$ together with isomorphisms

$$
\theta: m_{12}^{*} L \otimes m_{01}^{*} L \rightarrow m_{02}^{*} L
$$

and

$$
\eta: \mathcal{O}_{\mathcal{U}} \rightarrow \Delta^{*} L
$$

which satisfy the following conditions:

$$
\left(m_{013}^{*} \theta\right) \circ\left(m_{123}^{*} \theta \otimes 1_{m_{01}^{*} L}\right)=\left(m_{023}^{*} \theta\right) \circ\left(1_{m_{23}^{*} L} \otimes m_{012}^{*} \theta\right)
$$

as isomorphisms

$$
m_{23}^{*} L \otimes m_{12}^{*} L \otimes m_{01}^{*} L \rightarrow m_{03}^{*} L .
$$

Here we use

$$
m_{i j}: \mathcal{U} \times_{X} \mathcal{U} \times_{X} \mathcal{U} \times_{X} \mathcal{U} \rightarrow \mathcal{U} \times_{X} \mathcal{U}
$$

to denote the obvious maps.

$$
\left(1_{\mathcal{U}} \times \Delta\right)^{*} \theta \circ\left(m_{1}^{*} \eta \times 1_{L}\right)=r .
$$

$$
\left(\Delta \times 1_{\mathcal{U}}\right)^{*} \theta \circ\left(1_{L} \times m_{0}^{*} \eta\right)=l .
$$

In other words, a presentation of a gerbe on a twisted version of $X$ is a quadruple

$$
\left(f: \mathcal{U}^{1} \rightarrow \mathcal{U}^{1}, L \rightarrow \mathcal{U}^{1}, \theta, \eta\right)
$$

satisfying the above compatibilities.

We often write these as the isomorphisms

$$
\theta_{i j k}: L_{j k} \otimes f_{j k}^{*} L_{i j} \rightarrow L_{i k}
$$

and

which satisfy

$$
\eta_{i}: \mathcal{O} \rightarrow L_{i i}
$$

$$
\begin{gathered}
\theta_{i j l} \circ\left(\theta_{j k l} \otimes 1_{L_{i j}}\right)=\theta_{i k l} \circ\left(1_{L_{k l}} \otimes f_{k l}^{*} \theta_{i j k}\right), \\
\theta_{i j j} \circ\left(\eta_{j} \otimes 1_{L_{i j}}\right)=l_{i j},
\end{gathered}
$$

and

$$
\theta_{i i j} \circ\left(1_{L_{i j}} \otimes \eta_{j}\right)=r_{i j} .
$$

Definition 4.1. Two presentations ${ }_{(L, \theta, \eta)} X_{f}$ and ${ }_{\left(L^{\prime}, \theta^{\prime}, \eta^{\prime}\right)} X_{f^{\prime}}$ of gerbes on twisted versions of $X$ with respect to the atlas $\mathcal{U} \rightarrow X$ are said to be strongly equivalent if $f=f^{\prime}$, and there is an isomorphism $L \rightarrow L^{\prime}$ which intertwines $\theta$ with $\theta^{\prime}$ and $\eta$ with $\eta^{\prime}$. 
Finally, let us comment on the notion of weak equivalence of two presentations $(L, \theta, \eta) X_{f}$ and ${ }_{\left(L^{\prime}, \theta^{\prime}, \eta^{\prime}\right)} X_{f^{\prime}}$ of gerbes on twisted versions of $X$ over the same cover. Note that any equivalence $h$ of two twisted fibrations $X_{f}$ and $X_{f^{\prime}}$ gives rise to a map $\Xi(h)$ between presentations of gerbes on $X_{f^{\prime}}$ and presentations of gerbes on $X_{f}$. Guided by this observation we define a weak equivalence between ${ }_{(L, \theta, \eta)} X_{f}$ and ${ }_{\left(L^{\prime}, \theta^{\prime}, \eta^{\prime}\right)} X_{f^{\prime}}$ to be a triple $(h ; Q, \tau)$, where $h$ is an equivalence between $f$ and $f^{\prime}$, and the pair $(Q, \tau)$ is an equivalence between the two gerbe presentations $(L, \theta, \eta)$ and $\Xi(h)\left(L^{\prime}, \theta^{\prime}, \eta^{\prime}\right)$ on $X_{f}$. The map $\Xi(h)$ is defined on objects by

$$
\left(L^{\prime}, \theta^{\prime}, \eta^{\prime}\right) \mapsto\left(h_{1}^{*} L^{\prime}, h_{2}^{*} \theta^{\prime}, h_{0}^{*} \eta^{\prime}\right) .
$$

4.2. Classification of gerbes and the Leray-Serre spectral sequence. Isomorphism classes of gerbes on the space $X_{f}$ are classified by (the limit over covers $\mathfrak{C}$ of $X_{f}$ of) the second total cohomology group of the double complex

$$
\check{C}_{\mathfrak{C}}^{q}\left(\left(\mathcal{U}_{f}\right)^{p}, \mathcal{O}^{\times}\right)
$$

where $p \geq 0$ and $q \geq 0$. We point out that the open sets in $\mathfrak{C}$ are small and have nothing to do with $\mathcal{U}$ and $\mathcal{U}_{f}$. We will write this complex with $p$ increasing in the horizontal direction and $q$ increasing in the vertical direction. The rows only have cohomology in degree zero, so this double complex is equivalent to the kernel column of the first horizontal map: The column situated at $p=-1$, which is nothing but the Čech complex $\check{C}_{\mathfrak{C}}^{\bullet}\left(X_{f}, \mathcal{O}^{\times}\right)$on $X_{f}$, for the sheaf $\mathcal{O}^{\times}$. In other words, we have

$$
H^{2}\left(X_{f}, \mathcal{O}^{\times}\right) \cong \mathbb{H}^{2}\left(\check{C}_{\mathfrak{C}}^{\bullet}\left(\left(\mathcal{U}_{f}\right)^{\bullet}, \mathcal{O}^{\times}\right)\right) .
$$

The right hand side should be thought of as follows: We can think of a gerbe on $X_{f}$ as a gerbe on $\mathcal{U}_{f}=\left(\mathcal{U}_{f}\right)^{0}$ together with a twisted line bundle for the difference of the pullback gerbes on $\left(\mathcal{U}_{f}\right)^{1}$ (an isomorphism of the two pullback gerbes) and a relation between the pullback of these twisted line bundles to $\left(\mathcal{U}_{f}\right)^{2}$ which itself goes to the identity in $\left(\mathcal{U}_{f}\right)^{3}$. In fact the double complex $\check{C}_{\mathfrak{C}^{\mathcal{C}}}^{q}\left(\left(\mathcal{U}_{f}\right)^{p}, \mathcal{O}^{\times}\right)$is the $E_{0}$ term of the Leray-Serre spectral sequence which computes $H^{2}\left(X_{f}, \mathcal{O}^{\times}\right)$with respect to the map $\pi_{f}: X_{f} \rightarrow B$. We have

$$
\begin{gathered}
E_{f ; 0}^{p, q}=\check{C}_{\mathfrak{C}}^{q}\left(\left(\mathcal{U}_{f}\right)^{p}, \mathcal{O}^{\times}\right), \\
E_{f ; 1}^{p, q}=H^{q}\left(\left(\mathcal{U}_{f}\right)^{p}, \mathcal{O}^{\times}\right) \cong \check{C}_{\mathfrak{B}}^{p}\left(B, R^{q} \pi_{f *} \mathcal{O}^{\times}\right),
\end{gathered}
$$

and

$$
E_{f ; 2}^{p, q}=H^{p}\left(B, R^{q} \pi_{f *} \mathcal{O}^{\times}\right) .
$$

In other words, we think of the Leray-Serre spectral sequence for $\pi_{f}: X_{f} \rightarrow B$ as a spectral sequence associated with a "Čech to Čech" double complex where one of the covers is by big opens and one is by small opens.

So far everything we have said is general and applies to any fibration $c: W \rightarrow B$, where we would replace $\mathcal{U}_{f}^{\bullet}$ by the fiber products over $W$ of the disjoint union of the pullback by $c$ of a cover of the base.

Now the isomorphism of simplicial manifolds $\left(\mathcal{U}_{f}\right)^{\bullet} \cong\left(\mathcal{U}^{\bullet}\right)_{f}$ says that we can classify gerbes on $X_{f}$ through data defined on $X$; we have

$$
H^{2}\left(X_{f}, \mathcal{O}^{\times}\right) \cong \mathbb{H}^{2}\left(\check{C}_{\mathfrak{C}}^{\bullet}\left(\left(\mathcal{U}_{f}\right)^{\bullet}, \mathcal{O}^{\times}\right)\right) \cong \mathbb{H}^{2}\left(\check{C}_{\mathfrak{C}}^{\bullet}\left(\left(\mathcal{U}^{\bullet}\right)_{f}, \mathcal{O}^{\times}\right)\right) .
$$

Of course we will also use the isomorphism of simplicial manifolds $\left(\mathcal{U}_{f}\right)^{\bullet} \cong\left(\mathcal{U}^{\bullet}\right)_{f}$ from equation (4.2) to induce an isomorphism from the spectral sequence $\left(E_{f ; \bullet}, d_{\bullet}\right)$ 
to a new spectral sequence $\left(E_{\bullet}, d_{f ; \bullet}\right)$, where

$$
\begin{gathered}
E_{0}^{p, q}=\check{C}_{\mathfrak{C}^{q}}^{q}\left(\left(\mathcal{U}^{p}\right)_{f}, \mathcal{O}^{\times}\right), \\
E_{1}^{p, q}=H^{q}\left(\left(\mathcal{U}^{p}\right)_{f}, \mathcal{O}^{\times}\right) \cong \check{C}_{\mathfrak{B}}^{p}\left(B, R^{q} \pi_{f *} \mathcal{O}^{\times}\right),
\end{gathered}
$$

and

$$
E_{2}^{p, q}=E_{f ; 2}^{p, q}=H^{p}\left(B, R^{q} \pi_{f *} \mathcal{O}^{\times}\right) .
$$

Remark 4.2. Notice that the presentations of gerbes described in previous sections give classes in $\mathbb{H}^{2}\left(\check{C}^{\bullet}\left(\left(\mathcal{U}^{\bullet}\right)_{f}, \mathcal{O}^{\times}\right)\right) \cong H^{2}\left(X_{f}, \mathcal{O}^{\times}\right)$in the following manner. The line bundle $L$ is represented by an element of $\check{C}_{\mathfrak{C}}^{1}\left(\left(\mathcal{U}^{1}\right)_{f}, \mathcal{O}^{\times}\right)$, and the isomorphism $\theta$ is represented by an element of $\check{C}_{\mathfrak{C}}^{0}\left(\left(\mathcal{U}^{2}\right)_{f}, \mathcal{O}^{\times}\right)$.

\section{Reinterpretation AND DUALITy}

A fibration $X \rightarrow B$ defines a stack of abelian categories on $B$ by pushing forward the stack $\mathfrak{M o d}(X)$ from $X$. To an open set $U$ in $B$ we associate the stack of abelian categories $\operatorname{Mod}\left(\pi^{-1}(U)\right)$. On the other hand, given any gerbe over a twisted version of $X$, which trivializes along the pullback of an open cover of $B$, we can similarly associate a stack of abelian categories on $B$. Below, we explicitly re-express the data defining a presentation of a gerbe over a twisted version of $X$ in terms of the descent data of a stack of abelian categories on $B$, which is locally on the base given as the stack on $B$ which was associated to $X$. Of course, this new stack defined by this descent data is isomorphic to the stack of sheaves on the gerbe corresponding to the presentation.

Consider the sheaf of groupoids given by $\operatorname{Pic}(X / B) \times \operatorname{Aut}(X / B)$. The multiplication rule for this sheaf is

$$
\left(L_{1}, f_{1}\right)\left(L_{2}, f_{2}\right)=\left(L_{1} \otimes f_{1}^{*} L_{2}, f_{2} \circ f_{1}\right) .
$$

In these terms, a gerbe presentation on $X_{f}$ consists of isomorphisms

$$
\left(L_{j k}, f_{j k}\right)\left(L_{i j}, f_{i j}\right) \rightarrow\left(L_{i k}, f_{i k}\right)
$$

or

$$
\theta_{i j k}: L_{j k} \otimes f_{j k}^{*} L_{i j} \rightarrow L_{i k}
$$

and

$$
\eta_{i}:(\mathcal{O}, 1) \rightarrow\left(L_{i i}, 1\right)
$$

for which the diagram

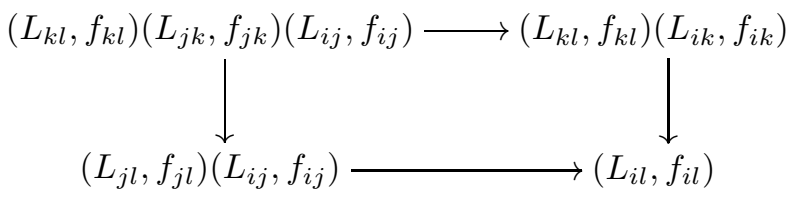

is commutative. Let

$$
A_{i j}: \operatorname{Mod}\left(\pi^{-1}\left(U_{i j}\right)\right) \rightarrow \operatorname{Mod}\left(\pi^{-1}\left(U_{i j}\right)\right)
$$

be the functor given on objects by

$$
A_{i j}(\mathcal{M})=L_{i j} \otimes f_{i j}^{*} \mathcal{M}
$$

We use $\boldsymbol{\theta}_{i j k}$ to denote the natural transformation

$$
\boldsymbol{\theta}_{i j k}: A_{j k} \circ A_{i j} \Rightarrow A_{i k} \text {. }
$$


Similarly, $\boldsymbol{\eta}_{i}$ will denote the natural transformation

$$
\boldsymbol{\eta}_{i}: 1_{i i} \Rightarrow T_{L_{i i}}=T_{L_{i i}} \circ f_{i i}^{*}=A_{i i} .
$$

Thus we have reinterpreted a presentation of a gerbe as a collection of functors and natural transformations satisfying some commutative diagrams.

Lemma 5.1. A presentation of a gerbe over a twisted version of $X \rightarrow B$, on the atlas $\mathcal{U} \rightarrow X$, defines a collection of functors $A_{i j}$ and natural transformations $\boldsymbol{\theta}_{i j k}$ and $\boldsymbol{\eta}_{i}$ as above, so that the following diagrams commute:
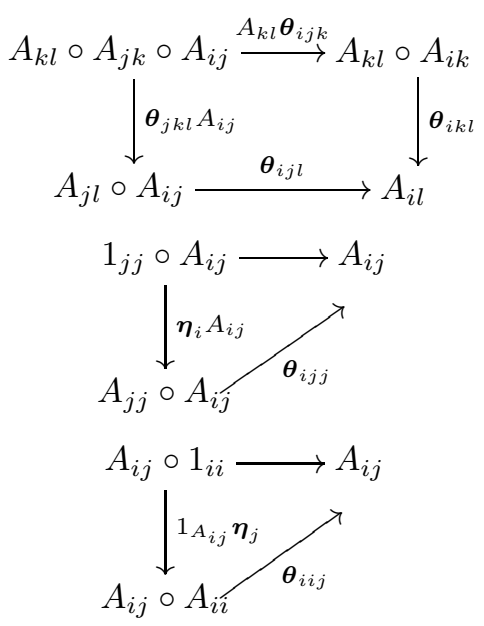

Proof. The commutativity of these diagrams follows from equations (4.3), (4.4) and (4.5). Indeed we need simply to observe that

$$
A_{k l} \boldsymbol{\theta}_{i j k}=1_{L_{k l}} \otimes f_{k l}^{*} \theta_{i j k}
$$

and

$$
\boldsymbol{\theta}_{j k l} A_{i j}=\theta_{j k l} \otimes 1_{L_{i j}} .
$$

Remark 5.2. Notice that if $(A, \boldsymbol{\theta}, \boldsymbol{\eta})$ are as above and $A^{\prime} \in A u t\left(\operatorname{Coh}\left(\mathcal{U}^{1}\right)\right)$ is a functor that is isomorphic to $A$ in a way compatible with restriction on a base, then there exist canonical natural transformations $\boldsymbol{\theta}^{\prime}$, and $\boldsymbol{\eta}^{\prime}$ such that $\left(A^{\prime}, \boldsymbol{\theta}^{\prime}, \boldsymbol{\eta}^{\prime}\right)$ satisfy the same diagrams as do $(A, \boldsymbol{\theta}, \boldsymbol{\eta})$. Therefore, we could have taken $A$ to be any automorphism of stacks isomorphic to $T_{L} \circ f^{*}$.

Definition 5.3. We call two triples $(A, \boldsymbol{\theta}, \boldsymbol{\eta})$ and $\left(A^{\prime}, \boldsymbol{\theta}^{\prime}, \boldsymbol{\eta}^{\prime}\right)$ strongly equivalent if there is a natural transformation between $A$ and $A^{\prime}$ which is compatible with restriction along the base, which intertwines $\boldsymbol{\theta}$ with $\boldsymbol{\theta}^{\prime}$, and also intertwines $\boldsymbol{\eta}$ with $\boldsymbol{\eta}^{\prime}$.

A weak equivalence of triples $(A, \boldsymbol{\theta}, \boldsymbol{\eta})$, and $\left(A^{\prime}, \boldsymbol{\theta}^{\prime}, \boldsymbol{\eta}^{\prime}\right)$ is given by a pair $(B, \boldsymbol{v})$, where $B: \operatorname{Mod}(\mathcal{U}) \rightarrow \operatorname{Mod}(\mathcal{U})$ is an autoequivalence and

$$
\boldsymbol{v}_{i j}: A_{i j} \circ B_{i} \Rightarrow B_{j} \circ A_{i j}^{\prime}
$$

is an invertible natural transformation which satisfies the equality of natural transformations

$$
\boldsymbol{v}_{i k} \circ\left(\boldsymbol{\theta}_{i j k} B_{i}\right)=\left(B_{k} \boldsymbol{\theta}_{i j k}^{\prime}\right) \circ\left(\boldsymbol{v}_{j k} A_{i j}^{\prime}\right) \circ\left(A_{j k} \boldsymbol{v}_{i j}\right)
$$


as natural transformations

$$
A_{j k} \circ A_{i j} \circ B_{i} \Rightarrow B_{k} \circ A_{i k}^{\prime} .
$$

Indeed, we can recognize equation (5.4) as containing the maps

$$
L_{i j} \otimes f_{i j}^{*} Q_{i} \rightarrow Q_{j} \otimes h_{j}^{*} L_{i j}^{\prime}
$$

Remark 5.4. Lemma 5.1 gives a fully faithful functor from the category whose objects are presentations ${ }_{(L, \theta, \eta)} X_{f}$ of gerbes on twisted versions of $X \rightarrow B$ using the atlas $\mathcal{U} \rightarrow X$ and whose morphisms are weak/strong equivalences, to the category whose objects are triples $(A, \boldsymbol{\theta}, \boldsymbol{\eta})$ and whose morphisms are weak/strong equivalences.

Remark 5.5. Any triple $(A, \boldsymbol{\theta}, \boldsymbol{\eta})$ defines an abelian category whose objects are sheaves $\mathcal{S}$ on $\mathcal{U}$, along with isomorphisms

$$
A\left(p_{0}^{*} \mathcal{S}\right) \rightarrow A\left(p_{1}^{*} \mathcal{S}\right) \quad \text { or } \quad A_{i j}\left(\mathcal{S}_{i}\right) \rightarrow \mathcal{S}_{j}
$$

such that
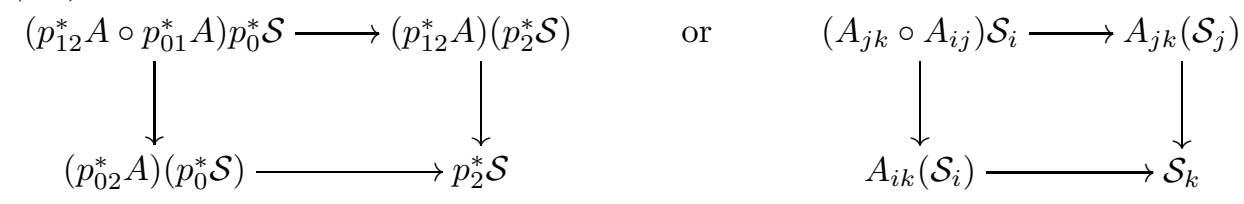

commutes, and morphisms are maps $\mathcal{S} \rightarrow \mathcal{T}$ such that the diagram

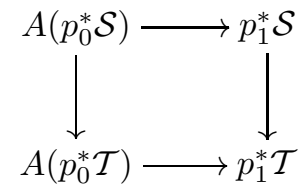

or

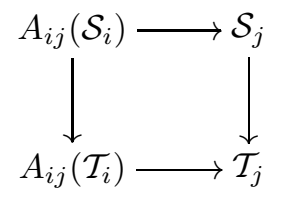

commutes. Any strong equivalence between triples $(A, \boldsymbol{\theta}, \boldsymbol{\eta})$ and $\left(A^{\prime}, \boldsymbol{\theta}^{\prime}, \boldsymbol{\eta}^{\prime}\right)$ induces an isomorphism of the associated abelian categories. If the triple $(A, \boldsymbol{\theta}, \boldsymbol{\eta})$ comes from a gerbe presentation ${ }_{(L, \theta, \eta)} X_{f}$, then the category we have described is isomorphic to the category of sheaves of weight $(-1)$ on the presentation ${ }_{(L, \theta, \eta)} X_{f}$. In the future, we will make this identification implicit.

Definition 5.6. Suppose we are given maps $\pi: X \rightarrow B$ and $\rho: Y \rightarrow B$, and an object $\mathcal{P}$ in $\mathrm{D}\left(X \times_{B} Y\right)$ giving a derived equivalence $\Phi: \mathrm{D}(Y) \rightarrow \mathrm{D}(X)$. We call a gerbe presentation $(L, \theta, \eta) X_{f} \Phi$-compatible if we have

$$
A \circ \Phi=\Phi \circ C
$$

where $A \in \operatorname{Aut}\left(\operatorname{Coh}\left(\mathcal{U}^{1}\right)\right)$ corresponds to ${ }_{(M, \theta, \eta)} X_{f}$ and $C \in \operatorname{Aut}\left(\operatorname{Coh}\left(\mathcal{V}^{1}\right)\right)$ is isomorphic to the functor $\mathcal{S} \rightarrow M \otimes g^{*} \mathcal{S}$, for some line bundle $M$, and an automorphism $g$ preserving the projection to the base.

We call a gerbe $\mathfrak{X} \rightarrow B$ over a twisted version of $X \Phi$-compatible if it admits a $\Phi$-compatible presentation ${ }_{(L, \theta, \eta)} X_{f}$. 
5.1. The dual gerbe on the dual fibration. Using our description of presentations of gerbes on twisted versions of $X$, we can now employ the base changed derived equivalence

$$
\Phi_{U^{1}}: \mathrm{D}(\mathcal{V}) \rightarrow \mathrm{D}(\mathcal{U})
$$

to map $\Phi$-compatible presentations of gerbes on twisted versions of $\pi: X \rightarrow B$ to $\Psi$-compatible presentations of gerbes on twisted versions of $\rho: Y \rightarrow B$, where we have chosen an inverse functor $\Psi: \mathrm{D}(Y) \rightarrow \mathrm{D}(X)$ to $\Phi$. It may help to keep in mind the commutative diagram

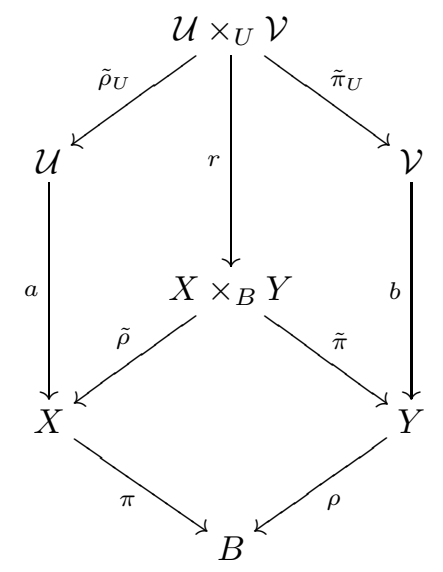

on which our presentations are based.

Consider the functor

$$
\Upsilon: \operatorname{Aut}\left(\operatorname{Coh}\left(\mathcal{U}^{1}\right)\right) \rightarrow \operatorname{Aut}\left(\mathrm{D}\left(\mathcal{V}^{1}\right)\right)
$$

given by

$$
\Upsilon: A \mapsto \Psi_{U^{1}} \circ A \circ \Phi_{U^{1}} .
$$

We will denote this on elements $A_{i j}$ by the equation

$$
\Upsilon\left(A_{i j}\right)=\Psi \circ A_{i j} \circ \Phi
$$

The functor $\Upsilon\left(A_{i j}\right)$ satisfies

$$
\Upsilon\left(A_{i j}\right) \cong T_{M_{i j}} \circ g_{i j}^{*}
$$

for some line bundles $M_{i j}$ on $\rho^{-1}\left(U_{i j}\right)$ and some fiber preserving automorphisms $g_{i j}$ of $\rho^{-1}\left(U_{i j}\right)$. Here the isomorphism respects the pullback along open sets in the base. In other words, this is an isomorphism of automorphisms of the pushforward of the stack of coherent sheaves on $Y$ to the base $B$.

Let $\operatorname{Aut}\left(\operatorname{Mod}\left(\mathcal{U}^{1}\right)\right)^{\Phi}$ be the sub group-groupoid of $\operatorname{Aut}\left(\operatorname{Mod}\left(\mathcal{U}^{1}\right)\right)$ consisting of the elements $A$ for which $\Upsilon(A)$ is isomorphic to $T_{M} \circ g^{*}$ for some line bundle $M$ and projection preserving automorphism $g$. We get an induced isomorphism of categories, where the morphisms are strong equivalences:

$$
\Upsilon: \operatorname{Aut}\left(\operatorname{Mod}\left(\mathcal{U}^{1}\right)\right)^{\Phi} \rightarrow \operatorname{Aut}\left(\operatorname{Mod}\left(\mathcal{V}^{1}\right)\right)^{\Psi} .
$$


By applying $\Upsilon$ to the diagram (5.1) we obtain the following commutative diagram:

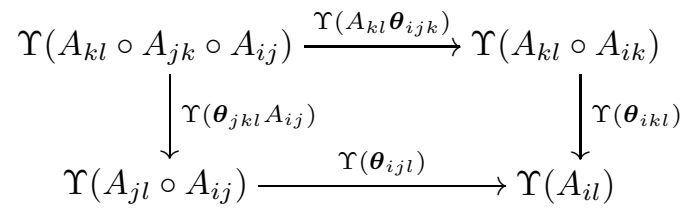

This is not quite what we want, but if we attach to this diagram three other commuting squares, we get the outer diagram below, which is the one that we want.

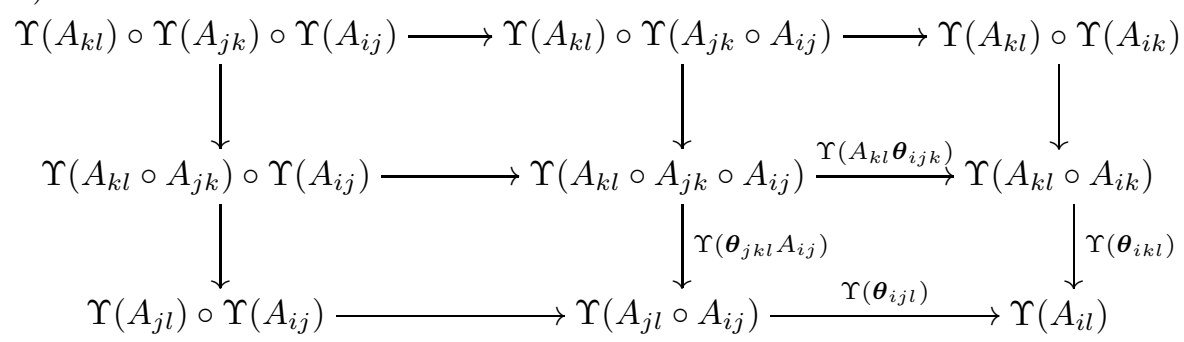

We now rewrite this, defining $\boldsymbol{T}_{i k l}$ to be the right hand side of the above diagram:

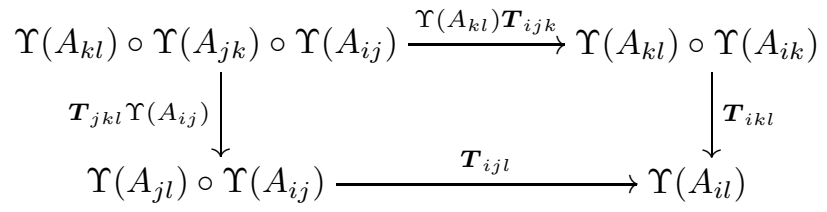

Similarly, by applying $\Upsilon$ to the diagrams (5.2) and (5.3) we get the diagrams

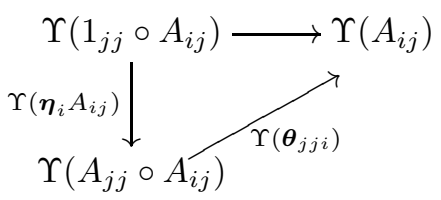

and

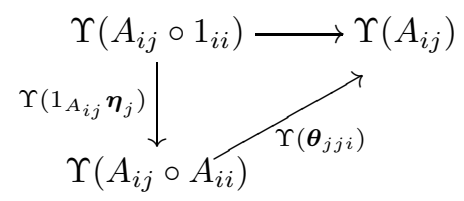

By applying $\Upsilon$ to the natural transformation $\boldsymbol{\eta}_{i}: 1_{i i} \Rightarrow A_{i i}$, we can define $\boldsymbol{\zeta}_{i}$ as the composition $1_{i i} \Rightarrow \Upsilon\left(1_{i i}\right) \Rightarrow \Upsilon\left(A_{i i}\right)$. Using $\boldsymbol{\zeta}_{i}: 1_{i i} \Rightarrow \Upsilon\left(A_{i i}\right)$ we can complete the above diagram as

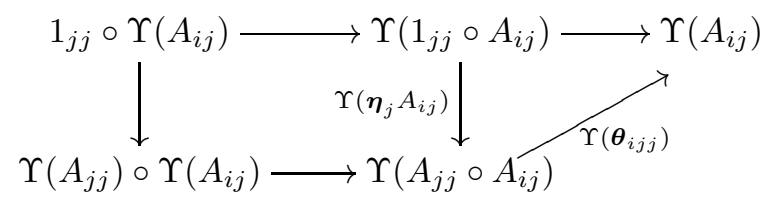


and

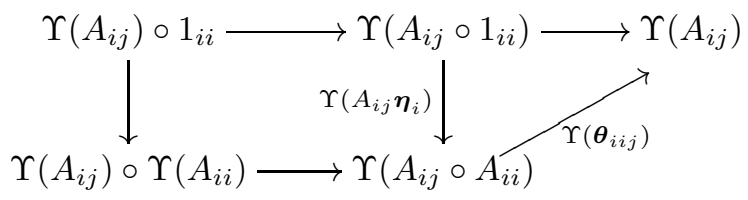

In other words, we obtain the commutative diagrams

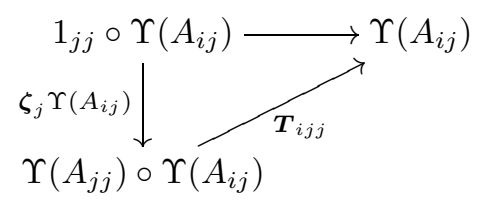

and

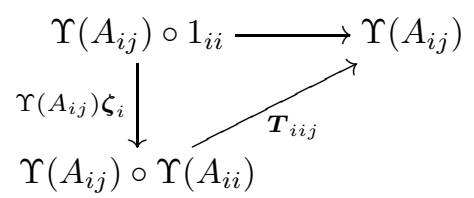

Notice that we end up with the same type of diagrams (5.9), (5.14), (5.15) that we started with on the other side (5.1), (5.2), (5.3). Finally, we apply the diagrams (5.9), (5.14), (5.15) to the structure sheaves of points $\mathbb{C}_{y}$ on $Y$ and to the structure sheaf $\mathcal{O}_{Y}$ of $Y$. The application to points shows that $g$ is a cocycle. Application to the structure sheaf gives a presentation of a gerbe on $Y_{g}$. This gives rise to a map

$$
b: \mathcal{V}=\coprod_{i} \rho^{-1}\left(U_{i}\right) \rightarrow Y,
$$

an automorphism $g: \mathcal{V}^{1} \rightarrow \mathcal{V}^{1}$, a line bundle $M \rightarrow \mathcal{V}^{1}$, and isomorphisms

$$
T_{i j k}: M_{j k} \otimes g_{j k}^{*} M_{i j} \rightarrow M_{i k}
$$

and

$$
\zeta_{i}: \mathcal{O} \rightarrow M_{i i},
$$

which satisfy

$$
\begin{gathered}
T_{i j l} \circ\left(T_{j k l} \otimes 1_{M_{i j}}\right)=T_{i k l} \circ\left(1_{M_{k l}} \otimes g_{k l}^{*} T_{i j k}\right), \\
T_{i j j} \circ\left(\zeta_{j} \otimes 1_{M_{i j}}\right)=l_{i j},
\end{gathered}
$$

and

$$
T_{i i j} \circ\left(1_{M_{i j}} \otimes \zeta_{j}\right)=r_{i j} .
$$

Thus, we have found a presentation $\left(g: \mathcal{V}^{1} \rightarrow \mathcal{V}^{1}, M \rightarrow \mathcal{V}^{1}, T, \zeta\right)$ of a gerbe on a twisted version of $Y$. This gerbe presentation ${ }_{(M, T, \zeta)} Y_{g} \rightarrow Y_{g}$ over a twisted version of $Y$ was found starting from the presentation of the gerbe ${ }_{(L, \theta, \eta)} X_{f} \rightarrow X_{f}$ over a twisted version of $X$. We state this as the following

Lemma 5.7. Given maps $\pi: X \rightarrow B$ and $\rho: Y \rightarrow B$ and an object $\mathcal{P} \in D\left(X \times{ }_{B} Y\right)$ giving a derived equivalence $\Phi: D(Y) \rightarrow D(X)$ and a quasi-inverse $\Psi$, there is a correspondence between $\Phi$-compatible presentations of gerbes on twisted versions of 
$X$ and $\Psi$-compatible presentations of gerbes on twisted versions of $Y$. Using strong equivalences as morphisms, this gives an isomorphism of categories.

Proof. Above.

Definition 5.8. Suppose $\mathfrak{X}$ is a $\Phi$-compatible gerbe on a twisted version of $X$ and $\mathfrak{Y}$ is the corresponding gerbe on a twisted version of $Y$ produced as described above. Then we say that $\mathfrak{Y}$ is $\Phi$-dual to $\mathfrak{X}$. $\mathfrak{Y}$ is defined by a $\Phi$-dual presentation $(M, T, \zeta) Y_{g}$ to a $\Phi$-compatible presentation ${ }_{(L, \theta, \eta)} X_{f}$ of $\mathfrak{X}$.

Notice that the definition of $T$ is encoded in the diagram

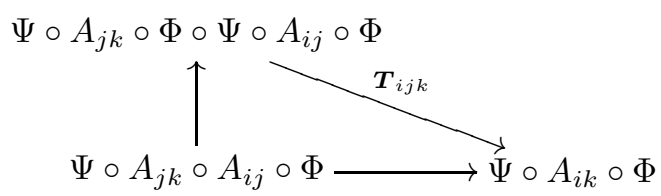

5.2. The new functor. It turns out that equations (5.6) and (5.19) secretly encode the description of a new functor. We have the following lemma.

Lemma 5.9. Suppose we are given compact, connected, complex manifolds $X$ and $Y$, maps $\pi: X \rightarrow B$ and $\rho: Y \rightarrow B$, where $\rho$ is flat, and an object

$$
\mathcal{P} \in \operatorname{Coh}\left(X \times{ }_{B} Y\right),
$$

flat over $Y$ giving a derived equivalence $\Phi: D_{c}^{b}(Y) \rightarrow D_{c}^{b}(X)$ and a quasi-inverse equivalence $\Psi$. Then for every $\Phi$-compatible gerbe presentation ${ }_{(L, \theta, \eta)} X_{f}$ and $\Phi$-dual gerbe presentation $(M, T, \zeta) Y_{g}$, we can define a functor

$$
\widetilde{\Phi}: D_{c}^{b}\left({ }_{(M, T, \zeta)} Y_{g},-1\right) \rightarrow D_{c}^{b}\left((L, \theta, \eta) X_{f},-1\right) .
$$

Proof. In order to see this, we must first manipulate equation (5.19) a little. By applying $\Phi$ on the left to the diagram (5.19) and attaching a commutative lower square, we get

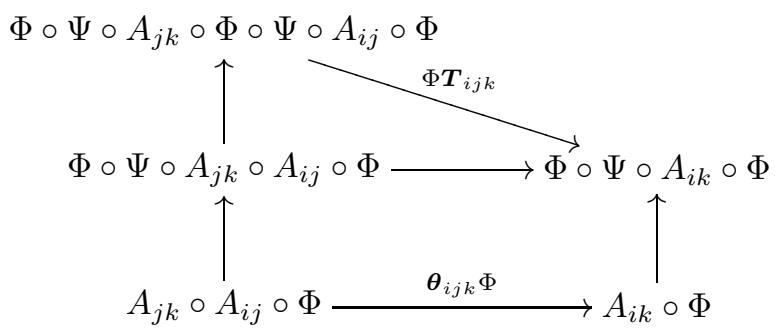

Taking the outer part of this we arrive at the commutative diagram

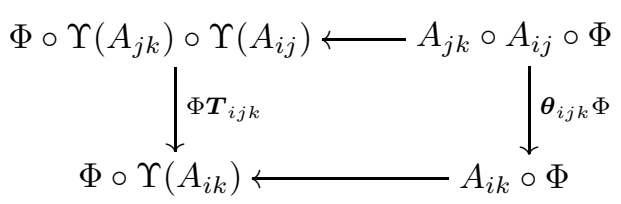


Remark 5.10. Every corner of this square is a functor implemented by a sheaf on the appropriate product. If we could find unique sheaf isomorphisms implementing the natural equivalences indicated by the arrows, this diagram would describe a sheaf of weight $(-1,1)$ on the product of our gerbe presentations, and it would be easy to show that such a sheaf implements an equivalence using the convolution product. However, it is not clear to the author how to produce these isomorphisms, and so we must follow a different path.

We start by defining a functor

$$
\widetilde{\phi}: \operatorname{Mod}\left({ }_{(M, T, \zeta)} Y_{g},-1\right) \rightarrow \operatorname{Mod}\left({ }_{(L, \theta, \eta)} X_{f},-1\right) .
$$

We will sometimes write this functor as

$$
\widetilde{\phi}\left(\mathcal{S}, \kappa: \Upsilon(A) p_{0}^{*} \mathcal{S} \rightarrow p_{1}^{*} \mathcal{S}\right)=(\widetilde{\phi}(\mathcal{S}), \widetilde{\phi}(\kappa)) .
$$

Here we define $\widetilde{\phi}(\mathcal{S})=\mathcal{H}^{0}\left(\Phi_{U}(\mathcal{S})\right)$, and $\widetilde{\phi}(\kappa): A\left(p_{0}^{*} \widetilde{\phi}(\mathcal{S})\right) \rightarrow p_{1}^{*} \widetilde{\phi}(\mathcal{S})$ is defined as the composition

$$
\begin{gathered}
A\left(p_{0}^{*}\left(\mathcal{H}^{0}\left(\Phi_{U}(\mathcal{S})\right)\right)\right) \rightarrow \mathcal{H}^{0}\left(A p_{0}^{*}\left(\Phi_{U}(\mathcal{S})\right)\right) \rightarrow \mathcal{H}^{0}\left(A\left(\Phi_{U^{1}} p_{0}^{*}(\mathcal{S})\right)\right) \\
\rightarrow \mathcal{H}^{0}\left(\Phi_{U^{1}}\left(\Upsilon(A) p_{0}^{*} \mathcal{S}\right)\right) \rightarrow \mathcal{H}^{0}\left(\Phi_{U^{1}}\left(p_{1}^{*} \mathcal{S}\right)\right) \rightarrow p_{1}^{*}\left(\mathcal{H}^{0}\left(\Phi_{U} \mathcal{S}\right)\right) .
\end{gathered}
$$

For the sake of sanity, we will change notation and skip the second and second to last term. We write the object of $\operatorname{Mod}\left({ }_{(M, T, \zeta)} Y_{g},-1\right)$ as

$$
\left(\left\{\mathcal{S}_{i}\right\},\left\{\kappa_{i j}: \Upsilon\left(A_{i j}\right) \mathcal{S}_{i} \rightarrow \mathcal{S}_{j}\right\}\right)
$$

It is sent by $\widetilde{\phi}$ to

$$
\left(\left\{\mathcal{H}^{0}\left(\Phi \mathcal{S}_{i}\right)\right\},\left\{\lambda_{i j}\right\}\right),
$$

where $\lambda_{i j}$ is defined by the composition

$$
\begin{gathered}
A_{i j} \mathcal{H}^{0}\left(\Phi \mathcal{S}_{i}\right) \rightarrow \mathcal{H}^{0}\left(A_{i j} \Phi \mathcal{S}_{i}\right) \rightarrow \mathcal{H}^{0}\left(\Phi \Upsilon\left(A_{i j}\right) \mathcal{S}_{i}\right) \rightarrow \mathcal{H}^{0}\left(\Phi \mathcal{S}_{j}\right), \\
\widetilde{\phi}\left(\left\{\mathcal{S}_{i}\right\},\left\{\kappa_{i j}\right\}\right)=\left(\left\{\mathcal{H}^{0}\left(\Phi \mathcal{S}_{i}\right)\right\},\left\{\lambda_{i j}\right\}\right) .
\end{gathered}
$$

The fact that this is an object of $\operatorname{Mod}\left({ }_{(M, T, \zeta)} Y_{g},-1\right)$ rests on the commutativity of the outer square in the following:

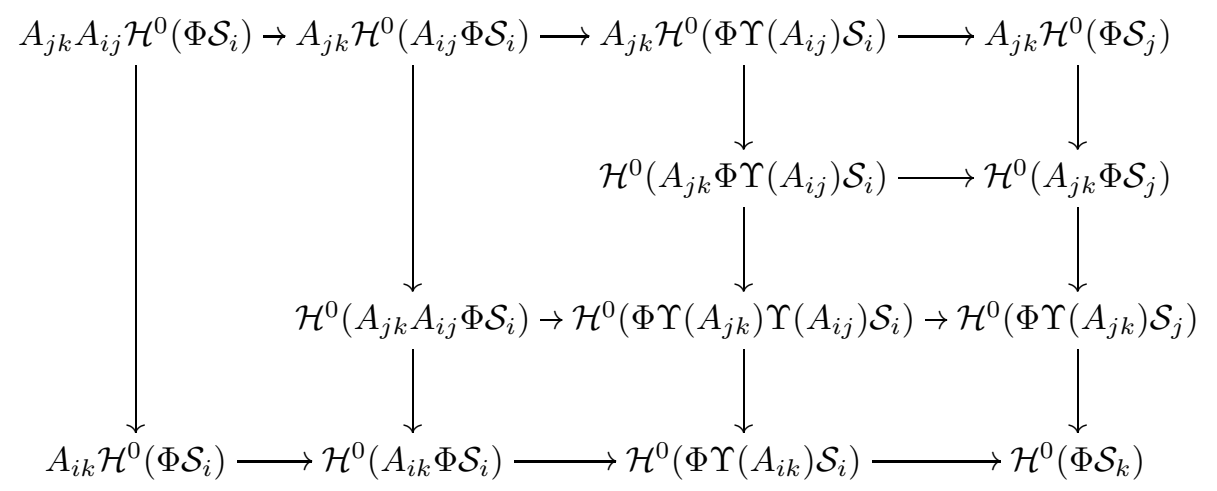

We can see that the outer square is commutative by checking that all the inner squares commute. The lower right square is commutative as a result of applying $\Phi$ followed by $\mathcal{H}^{0}$ to the descent diagram for $\left(\left\{\mathcal{S}_{i}\right\},\left\{\kappa_{i j}\right\}\right)$. This descent diagram can be found as diagram (5.5) where $A$ must be replaced by $\Upsilon(A)$. The square to the left of the lower right square is commutative using (5.21). The other squares of 
the diagram are clearly commutative. Therefore the functor $\widetilde{\phi}$ is well defined. We define $\widetilde{\Phi}$ to be the right derived functor $R \widetilde{\phi}$ of the left exact functor $\widetilde{\phi}$ :

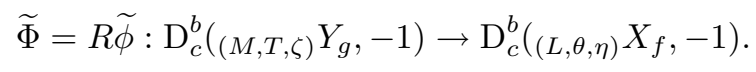

5.3. Twisted derived equivalences. In this section, we prove that the functor $\widetilde{\Phi}$ defined in Lemma 5.9 is actually an equivalence. We start by establishing some relationships between the pushforward of sheaves from an atlas and the relative Fourier-Mukai transform. Consider the diagram

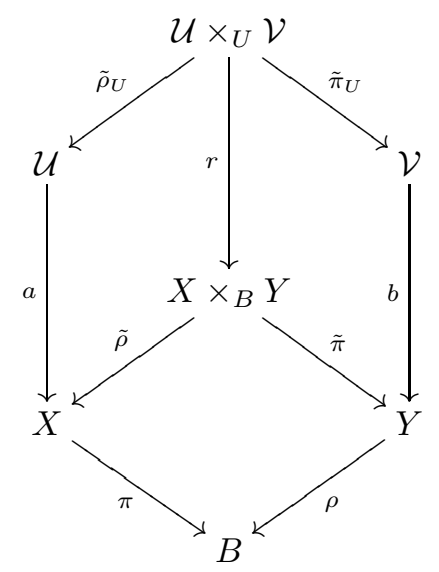

Then we have a natural equivalence of functors $\operatorname{Mod}(\mathcal{V}) \rightarrow \operatorname{Mod}(X)$ given by

$$
\phi \circ b_{*} \cong a_{*} \circ \phi_{U} .
$$

This implies that we have a natural equivalence of functors $\mathrm{D}(\mathcal{V}) \rightarrow \mathrm{D}(X)$ :

$$
\Phi \circ R b_{*} \cong R a_{*} \circ \Phi_{U} .
$$

Indeed note that for any object $\mathcal{S}$ in $\mathrm{D}(\mathcal{V})$ we have

$$
\Phi\left(R b_{*} \mathcal{S}\right)=R \tilde{\rho}_{*}\left(\tilde{\pi}^{*}\left(R b_{*} \mathcal{S}\right) \otimes \mathcal{P}\right) \cong R \tilde{\rho}_{*}\left(\left(R r_{*} \tilde{\pi}_{U}^{*} \mathcal{S}\right) \otimes \mathcal{P}\right) \cong R \tilde{\rho}_{*}\left(R r_{*}\left(\tilde{\pi}_{U}^{*} \mathcal{S} \otimes \mathcal{P}_{U}\right)\right)
$$

and

$$
R \tilde{\rho}_{*}\left(R r_{*}\left(\tilde{\pi}_{U}^{*} \mathcal{S} \otimes \mathcal{P}_{U}\right)\right) \cong R a_{*}\left(R \tilde{\rho}_{U *}\left(\tilde{\pi}_{U}^{*} \mathcal{S} \otimes \mathcal{P}_{U}\right)\right) \cong R a_{*} \Phi_{U}(\mathcal{S}) .
$$

Recall that our choice of presentations gives us maps $\mathfrak{a}=(a, L): \mathcal{U} \rightarrow \mathfrak{X}$ and $\mathfrak{b}=(b, M): \mathcal{V} \rightarrow \mathfrak{Y}$, fitting into the following commutative diagrams:

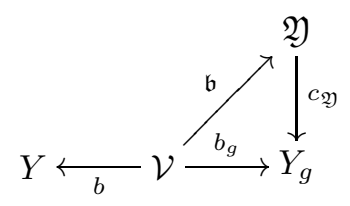

and

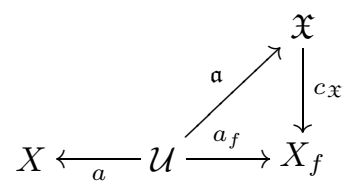


The pushforward functor $\mathfrak{a}_{*,-1}$ is defined as

$$
\begin{gathered}
\mathfrak{a}_{*,-1}: \operatorname{Mod}(\mathcal{U}) \rightarrow \operatorname{Mod}\left({ }_{(L, \theta, \eta)} X_{f},-1\right), \\
\mathfrak{a}_{*,-1}(\mathcal{S})=\left(p_{1 *}\left(A p_{0}^{*} \mathcal{S}\right), p_{12 *}\left(\boldsymbol{\theta}\left(p_{01}^{*} p_{0}^{*} \mathcal{S}\right)\right)\right) .
\end{gathered}
$$

We use the expression $p_{12 *}\left(\boldsymbol{\theta}\left(p_{01}^{*} p_{0}^{*} \mathcal{S}\right)\right)$ here as shorthand. Indeed recall that we have a natural transformation $\boldsymbol{\theta}: p_{12}^{*} A \circ p_{01}^{*} A \rightarrow p_{02}^{*} A$ and therefore an isomorphism of sheaves

$$
\boldsymbol{\theta}\left(p_{01}^{*} p_{0}^{*} \mathcal{S}\right):\left(p_{12}^{*} A\right)\left(p_{01}^{*}\left(A\left(p_{0}^{*} \mathcal{S}\right)\right)\right) \rightarrow\left(p_{02}^{*} A\right)\left(p_{01}^{*} p_{0}^{*} \mathcal{S}\right)
$$

We can use this to get a chain of isomorphisms

$$
\begin{gathered}
A p_{0}^{*} p_{1 *}\left(A p_{0}^{*} \mathcal{S}\right) \rightarrow p_{12 *}\left(\left(p_{12}^{*} A\right)\left(p_{01}^{*}\left(A\left(p_{0}^{*} \mathcal{S}\right)\right)\right)\right) \rightarrow p_{12 *}\left(\left(p_{02}^{*} A\right)\left(p_{01}^{*} p_{0}^{*} \mathcal{S}\right)\right) \\
\rightarrow p_{1}^{*} p_{1 *}\left(A p_{0}^{*} \mathcal{S}\right)
\end{gathered}
$$

as needed (see Remark 5.5). The associated derived functor is given by

$$
\begin{gathered}
R \mathfrak{a}_{*,-1}: \mathrm{D}(\mathcal{U}) \rightarrow \mathrm{D}\left({ }_{(L, \theta, \eta)} X_{f},-1\right), \\
R \mathfrak{a}_{*,-1}(\mathcal{S})=\left(R p_{1 *}\left(A p_{0}^{*} \mathcal{S}\right), R p_{12 *}\left(\boldsymbol{\theta}\left(p_{01}^{*} p_{0}^{*} \mathcal{S}\right)\right)\right) .
\end{gathered}
$$

Similarly, the pushforward functor $\mathfrak{b}_{*,-1}$ is defined as

$$
\begin{gathered}
\mathfrak{b}_{*,-1}: \operatorname{Mod}(\mathcal{V}) \rightarrow \operatorname{Mod}\left({ }_{(M, T, \zeta)} Y_{g},-1\right), \\
\mathfrak{b}_{*,-1}(\mathcal{T})=\left(q_{1 *}\left(\Upsilon(A) q_{0}^{*} \mathcal{T}\right), q_{12 *}\left(\boldsymbol{T}\left(q_{01}^{*} q_{0}^{*} \mathcal{T}\right)\right)\right),
\end{gathered}
$$

and its associated derived functor is given by

$$
\begin{gathered}
R \mathfrak{b}_{*,-1}: \mathrm{D}(\mathcal{V}) \rightarrow \mathrm{D}\left({ }_{(M, T, \zeta)} Y_{g},-1\right) \\
R \mathfrak{b}_{*,-1}(\mathcal{T})=\left(R q_{1 *}\left(\Upsilon(A) q_{0}^{*} \mathcal{T}\right), R q_{12 *}\left(\boldsymbol{T}\left(q_{01}^{*} q_{0}^{*} \mathcal{T}\right)\right)\right)
\end{gathered}
$$

Analogously to (5.22) , we have a natural equivalence of functors $\operatorname{Mod}(\mathcal{V}) \rightarrow$ $\operatorname{Mod}\left((L, \theta, \eta) X_{f}\right)$ given by

$$
\tilde{\phi} \circ \mathfrak{b}_{*,-1} \cong \mathfrak{a}_{*,-1} \circ \phi_{U},
$$

which induces the natural equivalence of functors $\mathrm{D}(\mathcal{V}) \rightarrow \mathrm{D}\left({ }_{(L, \theta, \eta)} X_{f}\right)$ given as

$$
\widetilde{\Phi} \circ R \mathfrak{b}_{*,-1} \cong R \mathfrak{a}_{*,-1} \circ \Phi_{U} .
$$

To see this, first note that applying the above to

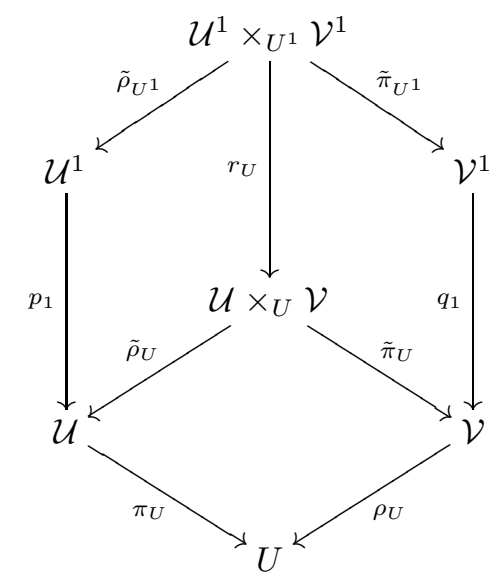

gives us

$$
\phi_{U} \circ q_{1 *} \cong p_{1 *} \circ \phi_{U^{1}}
$$


as functors $\operatorname{Mod}\left(\mathcal{V}^{1}\right) \rightarrow \operatorname{Mod}(\mathcal{U})$. Therefore, for any object $\mathcal{S}$ in $\operatorname{Mod}(\mathcal{V})$, we have

$$
\begin{gathered}
\widetilde{\phi}\left(\mathfrak{b}_{*,-1} \mathcal{S}\right)=\widetilde{\phi}\left(q_{1 *}\left(\Upsilon(A)\left(q_{0}^{*} \mathcal{S}\right)\right), q_{12 *}\left(T\left(q_{01}^{*} q_{0}^{*} \mathcal{S}\right)\right)\right), \\
\cong\left(\widetilde{\phi}\left(q_{1 *}\left(\Upsilon(A)\left(q_{0}^{*} \mathcal{S}\right)\right), \widetilde{\phi}\left(q_{12 *}\left(T\left(q_{01}^{*} q_{0}^{*} \mathcal{S}\right)\right)\right)\right)\right) .
\end{gathered}
$$

But recall that

$$
\widetilde{\phi}\left(q_{1 *}\left(\Upsilon(A)\left(q_{0}^{*} \mathcal{S}\right)\right)\right)=\mathcal{H}^{0}\left(\Phi_{U}\left(q_{1 *}\left(\Upsilon(A)\left(q_{0}^{*} \mathcal{S}\right)\right)\right)\right) \cong \phi_{U}\left(q_{1 *}\left(\Upsilon(A) q_{0}^{*} \mathcal{S}\right)\right) ;
$$

however,

$$
\phi_{U}\left(q_{1 *}\left(\Upsilon(A)\left(q_{0}^{*} \mathcal{S}\right)\right)\right) \cong p_{1 *}\left(\phi_{U^{1}}\left(\Upsilon(A)\left(q_{0}^{*} \mathcal{S}\right)\right)\right)
$$

and

$$
p_{1 *}\left(\phi_{U^{1}}\left(\Upsilon(A)\left(q_{0}^{*} \mathcal{S}\right)\right)\right) \cong p_{1 *}\left(A\left(\phi_{U^{1}} q_{0}^{*} \mathcal{S}\right)\right) \cong p_{1 *}\left(A p_{0}^{*}\left(\phi_{U} \mathcal{S}\right)\right) .
$$

Taking these isomorphisms into account, we also have

$$
\widetilde{\phi}\left(q_{12 *}\left(\boldsymbol{T}\left(q_{01}^{*} q_{0}^{*} \mathcal{S}\right)\right)\right) \cong p_{12 *}\left(\boldsymbol{\theta}\left(p_{01}^{*} p_{0}^{*}\left(\phi_{U} \mathcal{S}\right)\right)\right) ;
$$

this is essentially the content of diagram (5.8). Therefore,

$$
\widetilde{\phi}\left(\mathfrak{b}_{*,-1}(\mathcal{S})\right) \cong \mathfrak{a}_{*,-1}\left(\phi_{U}(\mathcal{S})\right),
$$

and taking derived functors, we have demonstrated the isomorphism (5.24).

Let $c: \mathfrak{S} \rightarrow S$ be a gerbe on a compact, connected, complex manifold $S$. We will need two facts about the triangulated categories $D_{c}^{b}(\mathfrak{S}, k)$, both of which were proven in [19].

- $D_{c}^{b}(\mathfrak{S}, k)$ admits a Serre functor defined by

$$
\begin{aligned}
\boldsymbol{S}_{\mathfrak{S}, k} & : D_{c}^{b}(\mathfrak{S}, k) \rightarrow D_{c}^{b}(\mathfrak{S}, k), \\
a & \mapsto a \otimes c^{*} \omega_{X}[n] .
\end{aligned}
$$

- The structure sheaves of points on $S$ viewed as sheaves of weight $k$ define a spanning class for $D_{c}^{b}(\mathfrak{S}, k)$.

We now recall a general criterion, due to Bondal-Orlov and Bridgeland, for a functor $\boldsymbol{F}: \mathscr{A} \rightarrow \mathscr{B}$ to be an equivalence of linear triangulated categories.

Theorem ([7, 8, 9]). Assume that $\mathscr{A}$ and $\mathscr{B}$ have Serre functors $\boldsymbol{S}_{\mathscr{A}}, \boldsymbol{S}_{\mathscr{B}}$, that $\mathscr{A} \neq 0, \mathscr{A}$ has a spanning class $C, \mathscr{B}$ is indecomposable, and that $\boldsymbol{F}: \mathscr{A} \rightarrow \mathscr{B}$ has left and right adjoints. Then $\boldsymbol{F}$ is an equivalence if

$$
\boldsymbol{F}: \operatorname{Hom}_{\mathscr{A}}^{i}\left(x_{1}, x_{2}\right) \underset{\rightarrow}{\longrightarrow} \operatorname{Hom}_{\mathscr{B}}^{i}\left(\boldsymbol{F} x_{1}, \boldsymbol{F} x_{2}\right), \quad \text { for all } i \in \mathbb{Z}, x_{1}, x_{2} \in C
$$

and it intertwines the Serre functors

$$
\boldsymbol{F} \circ \boldsymbol{S}_{\mathscr{A}}(x)=\boldsymbol{S}_{\mathscr{B}} \circ \boldsymbol{F}(x)
$$

on all elements $x \in C$ in the spanning class.

Theorem 5.11. Let $X$ and $Y$ be compact, connected, complex manifolds and $\pi$ : $X \rightarrow B$ and $\rho: Y \rightarrow B$ be maps of complex analytic spaces with $\rho$ flat and let $\mathcal{P} \in \operatorname{Coh}\left(X \times_{B} Y\right)$, which is flat over $Y$, implement a derived equivalence $\Phi$ : $D_{c}^{b}(Y) \rightarrow D_{c}^{b}(X)$. Let $\mathfrak{X}$ be a $\Phi$-compatible gerbe on a twisted version of $X$. Let $\mathfrak{Y}$ be a $\Phi$-dual gerbe to $\mathfrak{X}$ (so $\mathfrak{Y}$ lives over a twisted version of $Y$ ). Then we have an equivalence of categories

$$
D_{c}^{b}(\mathfrak{Y},-1) \rightarrow D_{c}^{b}(\mathfrak{X},-1) .
$$


Proof. First of all, note that our functor $\widetilde{\Phi}$ is a right-derived functor, and hence has a left adjoint. Since $\mathrm{D}_{c}^{b}(\mathfrak{Y},-1)$ and $\mathrm{D}_{c}^{b}(\mathfrak{X},-1)$ both have Serre functors, $\widetilde{\Phi}$ therefore has a right adjoint as well. It is obvious that $\mathrm{D}_{c}^{b}(\mathfrak{Y},-1)$ is not zero and that $\mathrm{D}_{c}^{b}(\mathfrak{X},-1)$ is indecomposable, and it has already been noted [19] that the points give a spanning class of $\mathrm{D}_{c}^{b}(\mathfrak{Y},-1)$.

Therefore, we simply need to show that $\widetilde{\Phi}$ is orthogonal on this spanning class and that it intertwines the Serre functors on this spanning class.

We claim that $\widetilde{\Phi}$ is orthogonal on the spanning class consisting of points in $Y_{g}$. Let $v_{1}$ and $v_{2}$ be any two points in $\mathcal{V}$. Then we know that

$$
\begin{aligned}
\operatorname{Hom}_{D_{c}^{b}(Y)}^{i}\left(R a_{*} \mathbb{C}_{v_{1}}, R a_{*} \mathbb{C}_{v_{2}}\right) \rightarrow \operatorname{Hom}_{D_{c}^{b}(X)}^{i}\left(\Phi R a_{*} \mathbb{C}_{v_{1}}, \Phi R a_{*} \mathbb{C}_{v_{2}}\right) \\
\cong \operatorname{Hom}_{D_{c}^{b}(X)}^{i}\left(R b_{*} \Phi_{U} \mathbb{C}_{v_{1}}, R b_{*} \Phi_{U} \mathbb{C}_{v_{2}}\right)
\end{aligned}
$$

is an isomorphism, where we have used the isomorphism (5.23). Now the commutative diagram

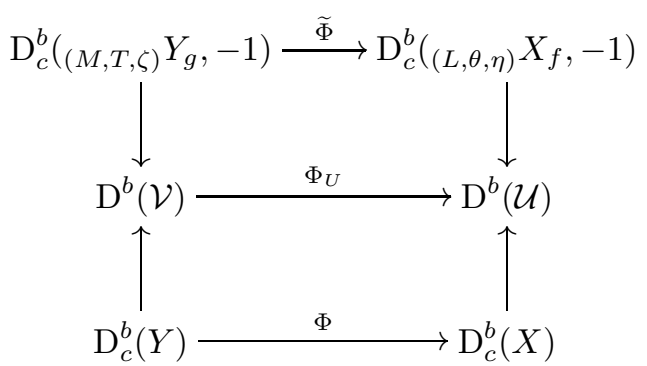

induces the following commutative diagram where the vertical arrows are isomorphisms, with inverses given by the various pushforward functors; see (5.23) and (5.24):

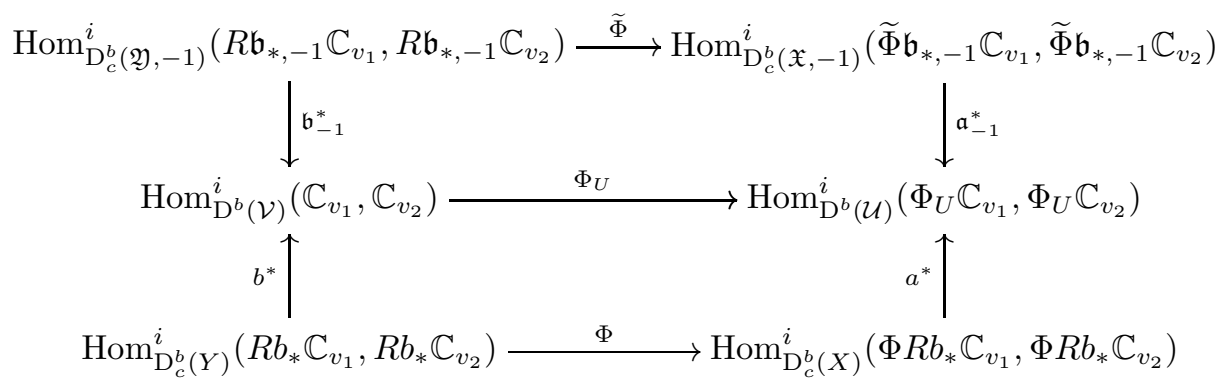

Since $\Phi$ is an equivalence, the bottom is an isomorphism, and so we are done. Also using the fact that $\Phi$ is an equivalence, we know that $\Phi$ intertwines the Serre functors of $\mathrm{D}_{c}^{b}(Y)$ and $\mathrm{D}_{c}^{b}(X)$. In other words,

$$
\Phi \circ \boldsymbol{S}_{Y} \cong \boldsymbol{S}_{X} \circ \Phi \text {. }
$$

Now pulling back by the cover $U \rightarrow B$ and using the property (3.2) we see that for all sheaves $\mathcal{S}$ on $\mathcal{V}$ we have

$$
\Phi_{U}\left(\left(b^{*} \omega_{Y}\right) \otimes \mathcal{S}[n]\right) \cong\left(a^{*} \omega_{X}\right) \otimes \Phi_{U}(\mathcal{S})[n] .
$$

Now we claim that $\widetilde{\Phi}$ intertwines the Serre functors of $D_{c}^{b}(\mathfrak{Y},-1)$ and $D_{c}^{b}(\mathfrak{X},-1)$ on the spanning class. Consider a point $v$ of $\mathcal{V}$. Observe that there is a sheaf $\mathcal{S}=\mathbb{C}_{v}$ with the properties $R b_{*} \mathcal{S} \cong \mathbb{C}_{b(v)}, R b_{g *} \mathcal{S} \cong \mathbb{C}_{b_{g}(v)}$, and $R \mathfrak{b}_{*,-1} \mathcal{S} \cong \mathbb{C}_{b_{\mathfrak{g}}(v),-1}$. Then 
if we let $\mathcal{T}=\Phi_{U}(\mathcal{S})$, we have $R a_{*} \mathcal{T} \cong \Phi\left(\mathbb{C}_{b(v)}\right)$ and $R \mathfrak{a}_{*,-1} \mathcal{T} \cong \widetilde{\Phi}\left(\mathbb{C}_{b_{g}(v),-1}\right)$. This follows from equations (5.23) and (5.24).

Moving forward, we have,

$$
\widetilde{\Phi}\left(\boldsymbol{S}_{\mathfrak{Y},-1}\left(\mathbb{C}_{b_{g}(v),-1}\right)\right)=\widetilde{\Phi}\left(\left(c_{\mathfrak{Y}}^{*} \omega_{Y_{g}}\right) \otimes \mathbb{C}_{b_{g}(v),-1}[n]\right) \cong \widetilde{\Phi}\left(c_{\mathfrak{Y}}^{*} \omega_{Y_{g}}\right) \otimes R \mathfrak{b}_{*,-1}(\mathcal{S})[n]
$$

and

$$
\widetilde{\Phi}\left(c_{\mathfrak{Y}}^{*} \omega_{Y_{g}}\right) \otimes R \mathfrak{b}_{g *,-1}(\mathcal{S})[n] \cong \widetilde{\Phi} R \mathfrak{b}_{*,-1}\left(b_{g}^{*} \omega_{Y_{g}} \otimes \mathcal{S}[n]\right)
$$

and by (5.24) and the fact that $\Phi$ intertwines the classical Serre functors (5.25),

$\widetilde{\Phi} R \mathfrak{b}_{*,-1}\left(b_{g}^{*} \omega_{Y_{g}} \otimes \mathcal{S}[n]\right) \cong R \mathfrak{a}_{*,-1} \Phi_{U}\left(\left(b^{*} \omega_{Y}\right) \otimes \mathcal{S}[n]\right) \cong R \mathfrak{a}_{*,-1}\left(\left(a^{*} \omega_{X}\right) \otimes \Phi_{U}(\mathcal{S})[n]\right)$.

However,

$R \mathfrak{a}_{*,-1}\left(\left(a^{*} \omega_{X}\right) \otimes \Phi_{U}(\mathcal{S})[n]\right) \cong R \mathfrak{a}_{f *,-1}\left(\left(a_{f}^{*} \omega_{X_{f}}\right) \otimes \mathcal{T}[n]\right) \cong\left(c_{\mathfrak{X}}^{*} \omega_{X_{f}}\right) \otimes R \mathfrak{a}_{*,-1} \mathcal{T}[n]$,

and finally

$$
\left(c_{\mathfrak{X}}^{*} \omega_{X_{f}}\right) \otimes R \mathfrak{a}_{*,-1} \mathcal{T}[n] \cong \boldsymbol{S}_{\mathfrak{X},-1}\left(\widetilde{\Phi}\left(\mathbb{C}_{b_{g}(v),-1}\right)\right) .
$$

Therefore, the Serre functors are intertwined on the spanning class by $\widetilde{\Phi}$, so we have checked all the criteria and hence $\widetilde{\Phi}$ is an equivalence.

5.4. Alternative method. In this subsection, we give another method for proving the equivalence. This method applies in different situations, allowing us to drop various coherence, smoothness, and compactness assumptions. We were convinced of the possibility/usefulness of such a method due to comments of D. Arinkin who kindly provided his insights to us after a reading of this paper. In the above, we have given an assignment $\mathbb{A}$ which takes a pair of shifted sheaves $\mathcal{P}, \mathcal{Q} \in \mathrm{D}_{c}\left(X \times_{B} Y\right)$ giving inverse equivalences, and a compatible gerbe presentation $\widetilde{X}={ }_{(L, \theta, \eta)} X_{f}$ to an equivalence $\mathrm{D}(\widetilde{Y}) \rightarrow \mathrm{D}(\widetilde{X})$, where $\widetilde{Y}$ is the dual presentation to $\widetilde{X}$ defined by the pair $(\mathcal{P}, \mathcal{Q})$. We encode this assignment as

$$
(\widetilde{X} ; \mathcal{P}, \mathcal{Q}) \rightsquigarrow[\mathbb{A}(\tilde{X} ; \mathcal{P}, \mathcal{Q}): \mathrm{D}(\widetilde{Y}(\tilde{X} ; \mathcal{P}, \mathcal{Q}),-1) \rightarrow \mathrm{D}(\tilde{X},-1)] .
$$

Observe that this assignment has the following properties:

- Given isomorphisms $\mathcal{P} \cong \mathcal{P}^{\prime}$ and $\mathcal{Q} \cong \mathcal{Q}^{\prime}$, the following diagram is commutative:

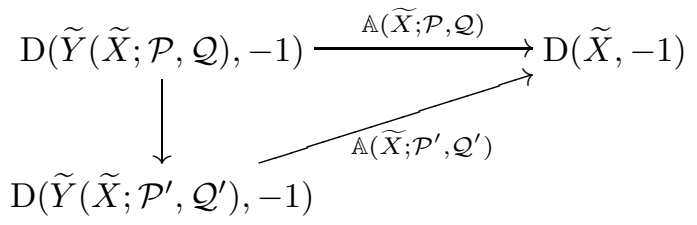

- Suppose we are given shifted coherent sheaves $\mathcal{P}_{1}, \mathcal{Q}_{1}$ on $X \times_{B} Y$, and $\mathcal{P}_{2}, \mathcal{Q}_{2}$ on $Y \times_{B} Z$ giving inverse equivalences, and a $\left(\mathcal{P}_{1}, \mathcal{Q}_{1}\right)$ compatible presentation $\tilde{X}$. Let $\widetilde{Y}$ denote $\tilde{Y}\left(\widetilde{X} ; \mathcal{P}_{1}, \mathcal{Q}_{1}\right)$, and suppose that $\widetilde{Y}$ is $\left(\mathcal{P}_{2}, \mathcal{Q}_{2}\right)$ compatible. Let $\widetilde{Z}$ denote $\widetilde{Z}\left(\widetilde{Y} ; \mathcal{P}_{2}, \mathcal{Q}_{2}\right)$. Then if $\mathcal{R}=\mathcal{P}_{1} * \mathcal{P}_{2}$ and $\mathcal{S}=$ $\mathcal{Q}_{2} * \mathcal{Q}_{1}$ are shifted sheaves, and we let

$$
\widetilde{Z}^{\prime}=\widetilde{Z}^{\prime}(\widetilde{X} ; \mathcal{R}, \mathcal{S}) \cong \widetilde{Z},
$$


the inverse equivalences given by $\mathcal{R}$ and $\mathcal{S}$ fit into the following commutative diagram:

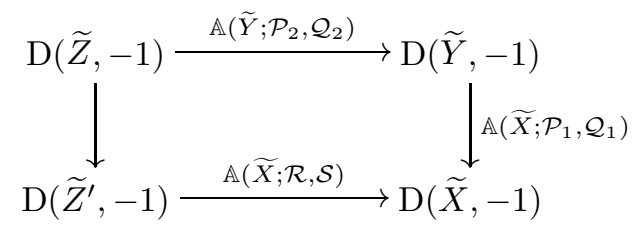

- If $Y=X, \mathcal{P}=\mathcal{Q}=\mathcal{O}_{\Delta}$, then $\tilde{Y}(\tilde{X} ; \mathcal{P}, \mathcal{Q}) \cong \tilde{X}$, and the resulting functor $\mathbb{A}\left(\widetilde{X} ; \mathcal{O}_{\Delta}, \mathcal{O}_{\Delta}\right): \mathrm{D}(\tilde{X}) \rightarrow \mathrm{D}(\tilde{X})$ is naturally equivalent to the identity.

These properties immediately imply the following:

Theorem 5.12. Let $X, Y, B$ be complex analytic spaces and $\pi: X \rightarrow B$ and $\rho: Y \rightarrow B$ be compact, flat maps. Let $\mathcal{P}, \mathcal{Q}$ be shifted coherent sheaves on $X \times_{B} Y$, flat over both factors, which implement inverse equivalences $\Phi: D(Y) \rightarrow D(X)$ and $\Psi: D(X) \rightarrow D(Y)$, and hence satisfy $\mathcal{P} * \mathcal{Q} \cong \mathcal{O}_{\Delta}$ and $\mathcal{Q} * \mathcal{P} \cong \mathcal{O}_{\Delta}$. Let $\mathfrak{X}$ be a gerbe on a twisted version of $X$, compatible with these equivalences. Let $\mathfrak{Y}$ be the dual gerbe to $\mathfrak{X}$, with respect to $\mathcal{P}$ and $\mathcal{Q}$ (so $\mathfrak{Y}$ lives over a twisted version of $Y$ ). Then we have an equivalences of categories

$$
D^{*}(\mathfrak{Y},-1) \rightarrow D^{*}(\mathfrak{X},-1)
$$

for $*=\emptyset, b$ and

$$
D_{c}^{*}(\mathfrak{Y},-1) \rightarrow D_{c}^{*}(\mathfrak{X},-1)
$$

for $*=b,-$.

Proof. By the above properties of $\mathbb{A}$, the functors associated to $\mathcal{P}$ and $\mathcal{Q}$ compose in both orders to functors equivalent to the identity, since $\mathcal{P}$ and $\mathcal{Q}$ convolve in both orders to sheaves isomorphic to the diagonals.

Remark 5.13. Using the definition of quasi-coherence found in 4], we expect that one would also get equivalences

$$
\mathrm{D}_{q c}^{*}(\mathfrak{Y},-1) \rightarrow \mathrm{D}_{q c}^{*}(\mathfrak{X},-1)
$$

for $*=b,-$.

\section{An APplication}

In this chapter we provide an application. We would like to emphasize that the geometric limitations we impose in the following are only necessary in order to allow for the setup of Donagi and Pantev to be reproduced via our setup. The situations that we will consider in the following are not the most general applications that one could imagine of our main theorem, even in the context of elliptic fibrations.

In the first section, we look at an analogue of Donagi and Pantev's setup for complex torus fibrations, and prove a general corollary of our main theorem. In the second section, we apply this corollary to prove their conjectures. 
6.1. Complex torus fibrations. Consider a complex torus fibration $X \rightarrow B$ with section $\sigma: B \rightarrow X$. By this we just mean that away from a closed co-dimension one analytic subset of $B$, the fiber of $\pi$ is a complex torus. Away from the singular fibers this is an analytic group space over $B$, using the section as an identity. Therefore over the complement of the singular fibers, the translation by a section (relative to $\sigma)$ defines an automorphism of the map $X \rightarrow B$.

Definition 6.1. Let us call a complex torus fibration with section $X \rightarrow B$ reasonable if $X$ is a compact, connected complex manifold, the map to $B$ is flat and if the translation by any local section $U \rightarrow X$ extends uniquely to an automorphism of the $\operatorname{map} X \times{ }_{B} U \rightarrow U$.

Let $\pi: X \rightarrow B$ and $\rho: Y \rightarrow B$ be reasonable complex torus fibrations with sections over a common base $B$. We use the section to give a group structure to the sheaves of sections. A sheaf $S$ on $X \times_{B} Y$ is called a bi-extension if for any local sections $\sigma: U \rightarrow X$ and $\psi: U \rightarrow Y$ we have that $\left.S\right|_{\sigma \times \rho^{-1}(U)}$ and $\left.S\right|_{\pi^{-1}(U) \times \psi}$ are line bundles and we have isomorphisms

$$
\left(p_{1}+p_{2}, p_{3}\right)^{*} S \cong p_{13}^{*} S \otimes p_{23}^{*} S
$$

on $X \times_{U} \sigma \times_{U} Y$ and isomorphisms

$$
\left(p_{1}, p_{2}+p_{3}\right)^{*} S \cong p_{12}^{*} S \otimes p_{13}^{*} S
$$

on $X \times_{U} Y \times_{U} \psi$ and that these isomorphisms satisfy certain natural compatibilities. In particular notice this implies that for small open sets $U \subset B$ and sections $\sigma$ of $X$ over $U$, and $\psi$ of $Y$ over $U$, if $f$ (and $g$ ) represent the translation action of $\sigma$ on $X(\psi$ on $Y)$ we have

$$
\left.f^{*}\left(\left.S\right|_{\pi^{-1}(U) \times \psi}\right) \cong S\right|_{\pi^{-1}(U) \times \psi} \otimes\left(\pi^{*}\left(\left.(\sigma, \psi)^{*} S\right|_{\pi^{-1}(U) \times \rho^{-1}(U)}\right)\right) .
$$

Now if $U$ has no non-trivial line bundles, then the line bundle on $U$ given by $\left(\left.(\sigma, \psi)^{*} S\right|_{\pi^{-1}(U) \times \rho^{-1}(U)}\right)$ can be trivialized, so we get an isomorphism

$$
\left.f^{*}\left(\left.S\right|_{\pi^{-1}(U) \times \psi}\right) \cong S\right|_{\pi^{-1}(U) \times \psi}
$$

Similarly there exists an isomorphism,

$$
\left.g^{*}\left(\left.S\right|_{\sigma \times \rho^{-1}(U)}\right) \cong S\right|_{\sigma \times \rho^{-1}(U)}
$$

Furthermore the bi-extension structure gives us isomorphisms

$$
(f \times 1)^{*}\left(\left.S\right|_{\pi^{-1}(U) \times \rho^{-1}(U)}\right) \cong S \otimes \tilde{\pi}^{*}\left(\left.S\right|_{\sigma \times \rho^{-1}(U)}\right)
$$

and

$$
(1 \times g)^{*}\left(\left.S\right|_{\pi^{-1}(U) \times \rho^{-1}(U)}\right) \cong S \otimes \tilde{\rho}^{*}\left(\left.S\right|_{\pi^{-1}(U) \times \psi}\right) .
$$

Suppose that $\pi: X \rightarrow B$ and $\rho: Y \rightarrow B$ are reasonable complex torus fibrations with section over the same base $B$ and let us denote by $\mathcal{X}$ and $\mathcal{Y}$ the sheaves of sections of $X$ and $Y$ respectively. By the above injections of sheaves, the sheaves $\mathcal{X}$ and $\mathcal{Y}$ become sub-sheaves of groups in $A u t_{X / B}$ and $A u t_{Y / B}$ respectively. We assume that there is a coherent Poincaré sheaf $\mathcal{P}$ living on $X \times_{B} Y$, flat over $Y$, that implements an equivalence of derived categories $\Phi: \mathrm{D}_{c}^{b}(Y) \rightarrow \mathrm{D}_{c}^{b}(X)$ and is a bi-extension. Consider two elements $\alpha \in H^{1}(B, \mathcal{Y})$ and $\beta \in H^{1}(B, \mathcal{X})$. Let $f \in \check{Z}^{1}\left(B, A u t_{X / B}\right)$ and $g \in \check{Z}^{1}\left(B, A u t_{Y / B}\right)$ be the automorphisms given by translation by some representatives for $\beta$ and $\alpha$ respectively. We now define a map

$$
\mathcal{X} \rightarrow R^{1} \rho_{\alpha *} \mathcal{O}^{\times}
$$


Indeed we can send a local section $\gamma_{i}$ over $U_{i}$ to the equivalence class

$$
\left[\left.\mathcal{P}\right|_{\gamma_{i} \times \rho^{-1}\left(U_{i}\right)}\right] \in H^{1}\left(\rho^{-1}\left(U_{i}\right), \mathcal{O}^{\times}\right) .
$$

Let $g_{i j}$ be the automorphism of $\rho^{-1}\left(U_{i j}\right)$ corresponding to the section $\alpha_{i j}$. Now, because of equation (6.2) (following from the reasonable nature of the torus fibrations and the bi-extension property of $\mathcal{P}$ ) we have, for sections $\gamma_{i}$ and $\gamma_{j}$ agreeing on the overlap,

$$
g_{i j}^{*}\left[\left.\mathcal{P}\right|_{\gamma_{i} \times \rho^{-1}\left(U_{i}\right)}\right]=\left[\left.\mathcal{P}\right|_{\gamma_{j} \times \rho^{-1}\left(U_{i}\right)}\right]
$$

in $H^{1}\left(\rho^{-1}\left(U_{i j}\right), \mathcal{O}^{\times}\right)$. This shows that the map we have described is well defined. Similarly, we have a map

$$
\mathcal{Y} \rightarrow R^{1} \pi_{\beta *} \mathcal{O}^{\times} .
$$

By taking the induced map on cohomology, we denote by $S_{\alpha}(\beta)$ the image of $\beta$ under the map

$$
H^{1}(B, \mathcal{X}) \rightarrow H^{1}\left(B, R^{1} \rho_{\alpha *} \mathcal{O}^{\times}\right) .
$$

Similarly, we denote by $S_{\beta}(\alpha)$ the image of $\alpha$ under the map

$$
H^{1}(B, \mathcal{Y}) \rightarrow H^{1}\left(B, R^{1} \pi_{\beta *} \mathcal{O}^{\times}\right) .
$$

Let us further assume that the images of $S_{\alpha}(\beta)$ and $S_{\beta}(\alpha)$ (via the differentials $d_{g ; 2}$ and $d_{f ; 2}$ described in Section 4.2) vanish in $H^{3}\left(B, \mathcal{O}^{\times}\right)$and that the image of $H^{2}\left(B, \mathcal{O}^{\times}\right)$vanishes in both $H^{2}\left(X_{\beta}, \mathcal{O}^{\times}\right)$and $H^{2}\left(X_{\alpha}, \mathcal{O}^{\times}\right)$. Then, according to the spectral sequence for the fibrations we find unique gerbes ${ }_{\alpha} X_{\beta} \rightarrow X_{\beta}$ and ${ }_{\beta} Y_{\alpha} \rightarrow Y_{\alpha}$ which correspond to $S_{\beta}(\alpha)$ and $S_{\alpha}(\beta)$ respectively. We then have the following corollary of the main theorem. For certain elliptic fibrations $X=Y$ with some possible further restrictions on the pair $(\beta, \alpha)$, this fact has already been proven by Donagi and Pantev [19]. For some other cases of $\beta$ and $\alpha$ this was conjectured. The following corollary proves the conjectures (see e.g. Conjecture 2.19) from [19, and as well it gives an alternative proof of the main results in the paper [19].

Corollary 6.2. In the above circumstance, we have

$$
D_{c}^{b}\left({ }_{-\beta} Y_{\alpha}, 1\right) \cong D_{c}^{b}\left({ }_{\alpha} X_{\beta}, 1\right) .
$$

Proof. In order to prove this we simply need to find gerbe presentations ${ }_{(L, \theta, \eta)} X_{f}$ of ${ }_{-\alpha} X_{\beta}$ and ${ }_{(M, T, \zeta)} Y_{g}$ of ${ }_{\beta} Y_{\alpha}$ which are dual in the sense of the main theorem. For then we will have

$$
\begin{aligned}
\mathrm{D}_{c}^{b}\left({ }_{-\beta} Y_{\alpha}, 1\right) & \cong \mathrm{D}_{c}^{b}\left((M, T, \zeta)_{{ }_{g}},-1\right) \cong \mathrm{D}_{c}^{b}\left({ }_{(L, \theta, \eta)} X_{f},-1\right) \\
& \cong \mathrm{D}_{c}^{b}\left({ }_{-\alpha} X_{\beta},-1\right) \cong \mathrm{D}_{c}^{b}\left({ }_{\alpha} X_{\beta}, 1\right)
\end{aligned}
$$

We do this simply by picking a reasonable choice for ${ }_{(L, \theta, \eta)} X_{f}$ and showing that its dual presentation represents ${ }_{\beta} Y_{\alpha}$.

We know that the gerbe ${ }_{-\alpha} X_{\beta}$ comes from the $E_{\infty}$ term of the Leray-Serre spectral sequence given by

$$
\left[\left[\left.\mathcal{P}\right|_{\left(\mathcal{U}^{1}\right)_{f} \times-\alpha}\right]\right] \in \frac{\left.\operatorname{ker}\left(H^{1}\left(\left(\mathcal{U}^{1}\right)_{f}, \mathcal{O}^{\times}\right)\right) \rightarrow H^{1}\left(\left(\mathcal{U}^{2}\right)_{f}, \mathcal{O}^{\times}\right)\right)}{\left.\operatorname{im}\left(H^{1}\left(\left(\mathcal{U}^{0}\right)_{f}, \mathcal{O}^{\times}\right)\right) \rightarrow H^{1}\left(\left(\mathcal{U}^{1}\right)_{f}, \mathcal{O}^{\times}\right)\right)} \cong H^{1}\left(B, R^{1} \pi_{\beta *} \mathcal{O}^{\times}\right) .
$$

In other words, $\left[\left[\left.\mathcal{P}\right|_{\left(\mathcal{U}^{1}\right)_{f} \times-\alpha}\right]\right]$ goes to zero in $H^{3}\left(B, \mathcal{O}^{\times}\right)$. The double brackets refer to the fact that $\left[\left[\left.\mathcal{P}\right|_{\left(\mathcal{U}^{1}\right)_{f} \times-\alpha}\right]\right]$ comes from the element $\left[\left.\mathcal{P}\right|_{\left(\mathcal{U}^{1}\right)_{f} \times-\alpha}\right] \in$ $H^{1}\left(\left(\mathcal{U}^{1}\right)_{f}, \mathcal{O}^{\times}\right)$. Concretely, we can see that

$$
\left[\left.\mathcal{P}\right|_{\left(\mathcal{U}^{1}\right)_{f} \times-\alpha}\right] \in \operatorname{ker}\left[H^{1}\left(\left(\mathcal{U}^{1}\right)_{f}, \mathcal{O}^{\times}\right) \rightarrow H^{1}\left(\left(\mathcal{U}^{2}\right)_{f}, \mathcal{O}^{\times}\right)\right]
$$


by the equation

$$
\begin{gathered}
{\left[\left.\mathcal{P}\right|_{\left(\mathcal{U}^{1}\right)_{f} \times-\alpha_{j k}}\right] \otimes f_{j k}^{*}\left[\left.\mathcal{P}\right|_{\left(\mathcal{U}^{1}\right)_{f} \times-\alpha_{i j}}\right]=\left[\left.\mathcal{P}\right|_{\left(\mathcal{U}^{1}\right)_{f} \times-\alpha_{j k}}\right] \otimes\left[\left.\mathcal{P}\right|_{\left(\mathcal{U}^{1}\right)_{f} \times-\alpha_{i j}}\right]} \\
=\left[\left.\mathcal{P}\right|_{\left(\mathcal{U}^{1}\right)_{f} \times\left(-\alpha_{i j}-\alpha_{j k}\right)}\right]=\left[\left.\mathcal{P}\right|_{\left(\mathcal{U}^{1}\right)_{f} \times-\alpha_{i k}}\right],
\end{gathered}
$$

where we have used equation (6.1). Consider the natural filtration

$$
0 \subset F^{2} \mathbb{H}^{2}\left(\check{C}^{\bullet}\left(\left(\mathcal{U}^{\bullet}\right)_{f}, \mathcal{O}^{\times}\right)\right) \subset F^{1} \mathbb{H}^{2}\left(\check{C}^{\bullet}\left(\left(\mathcal{U}^{\bullet}\right)_{f}, \mathcal{O}^{\times}\right)\right) \subset \mathbb{H}^{2}\left(\check{C}^{\bullet}\left(\left(\mathcal{U}^{\bullet}\right)_{f}, \mathcal{O}^{\times}\right)\right) .
$$

Here $F^{1} \mathbb{H}^{2}\left(\check{C}^{\bullet}\left(\left(\mathcal{U}^{\bullet}\right)_{f}, \mathcal{O}^{\times}\right)\right)$are the classes representable by an element whose component in $\check{C}^{2}\left(\left(\mathcal{U}^{0}\right)_{f}, \mathcal{O}^{\times}\right)$is trivial, and $F^{2} \mathbb{H}^{2}\left(\check{C}^{\bullet}\left(\left(\mathcal{U}^{\bullet}\right)_{f}, \mathcal{O}^{\times}\right)\right)$are the classes representable by an element whose components in $\check{C}^{2}\left(\left(\mathcal{U}^{0}\right)_{f}, \mathcal{O}^{\times}\right)$and $\check{C}^{1}\left(\left(\mathcal{U}^{1}\right)_{f}, \mathcal{O}^{\times}\right)$ are trivial. The map

$$
F^{1} \mathbb{H}^{2}\left(\check{C}^{\bullet}\left(\left(\mathcal{U}^{\bullet}\right)_{f}, \mathcal{O}^{\times}\right)\right) \rightarrow E_{\infty}^{1,1}
$$

sends an element $(L, \theta) \in \check{C}^{1}\left(\left(\mathcal{U}^{1}\right)_{f}, \mathcal{O}^{\times}\right) \times \check{C}^{0}\left(\left(\mathcal{U}^{2}\right)_{f}, \mathcal{O}^{\times}\right)$to the image in $E_{\infty}^{1,1}$ of the obvious modification $L^{\prime} \in \check{C}^{1}\left(\left(\mathcal{U}^{1}\right)_{f}, \mathcal{O}^{\times}\right)$of $L$, given by choosing an element in $\check{C}^{0}\left(\left(\mathcal{U}^{1}\right)_{f}, \mathcal{O}^{\times}\right)$which trivializes $\theta$. Notice that we have just replaced $L$ by an isomorphic line bundle $L^{\prime}$, in other words, $[L]=\left[L^{\prime}\right] \in H^{1}\left(\left(\mathcal{U}^{1}\right)_{f}, \mathcal{O}^{\times}\right)$. In the other direction consider the following set theoretic splitting of the surjection:

$$
F^{1} \mathbb{H}^{2}\left(\check{C}^{\bullet}\left(\left(\mathcal{U}^{\bullet}\right)_{f}, \mathcal{O}^{\times}\right)\right) \rightarrow E_{\infty}^{1,1} .
$$

Given an element $[[L]] \in E_{\infty}^{1,1}$, we know that $[L]$ goes to zero in $H^{1}\left(\left(\mathcal{U}^{2}\right)_{f}, \mathcal{O}^{\times}\right)$, and therefore the image of $L$ in $\check{C}^{1}\left(\left(\mathcal{U}^{2}\right)_{f}, \mathcal{O}^{\times}\right)$is bounded by an element $\theta$ in $\check{C}^{0}\left(\left(\mathcal{U}^{2}\right)_{f}, \mathcal{O}^{\times}\right)$. Since $[[L]]$ survives to $E_{3}^{1,1}=E_{\infty}^{1,1}, \theta$ goes to the trivial element in $\check{C}^{0}\left(\left(\mathcal{U}^{3}\right)_{f}, \mathcal{O}^{\times}\right)$. Therefore, the pair $(L, \theta)$ gives an element in $F^{1} \mathbb{H}^{2}\left(\check{C}^{\bullet}\left(\left(\mathcal{U}^{\bullet}\right)_{f}, \mathcal{O}^{\times}\right)\right)$. Because we are assuming that $F^{2} \mathbb{H}^{2}\left(\check{C}^{\bullet}\left(\left(\mathcal{U}^{\bullet}\right)_{f}, \mathcal{O}^{\times}\right)\right)$is trivial (the pullback of a gerbe from the base is trivial), the inverse map (the set theoretic splitting) is actually a group homomorphism, and we have written explicitly the two maps in the isomorphism $F^{1} \mathbb{H}^{2}\left(\check{C}^{\bullet}\left(\left(\mathcal{U}^{\bullet}\right)_{f}, \mathcal{O}^{\times}\right)\right) \cong E_{\infty}^{1,1}$.

We now apply the above in the case that $L=\left.\mathcal{P}\right|_{\left(\mathcal{U}^{1}\right)_{f} \times-\alpha}$; in other words $L_{i j}=$ $\left.\mathcal{P}\right|_{\left(\mathcal{U}^{1}\right)_{f} \times-\alpha_{i j}}$. We have canonical trivializations $\eta_{i}: \mathcal{O} \rightarrow L_{i i}$, and therefore we have produced a presentation of a gerbe ${ }_{(L, \theta, \eta)} X_{f}$ on $X$. Now the gerbe presentation $(L, \theta, \eta) X_{f}$ corresponds, according to the proof of Lemma [5.1, to the element

$$
T_{\left.\mathcal{P}\right|_{\left(\mathcal{U}^{1}\right)_{f} \times-\alpha}} \circ f^{*} \in \operatorname{Aut}\left(\operatorname{Coh}\left(\mathcal{U}^{1}\right)\right) .
$$

Pre-composing this by the Fourier-Mukai transform

$$
\Phi_{\mathcal{P}}: \mathrm{D}\left(\mathcal{V}^{1}\right) \rightarrow \mathrm{D}\left(\mathcal{U}^{1}\right)
$$

yields

$$
T_{\left.\mathcal{P}\right|_{\left(\mathcal{U}^{1}\right)_{f} \times-\alpha}} \circ f^{*} \circ \Phi_{\mathcal{P}} .
$$

Now using equations (3.4) and (6.3) we have

$$
f^{*} \circ \Phi_{\mathcal{P}} \cong \Phi_{(f \times 1)^{*} \mathcal{P}} \cong \Phi_{\mathcal{P} \otimes \tilde{\pi}^{*}\left(\left.\mathcal{P}\right|_{\beta \times \mathcal{V}^{1}}\right)} \cong \Phi_{\mathcal{P}} \circ T_{\left.\mathcal{P}\right|_{\beta \times \mathcal{V}^{1}}} .
$$

Now we have, using equations (3.5) and (6.4),

$$
T_{\left.\mathcal{P}\right|_{\left(\mathcal{U}^{1}\right)_{f} \times-\alpha}} \circ \Phi_{\mathcal{P}} \cong \Phi_{\tilde{\rho}^{*}\left(\left.\mathcal{P}\right|_{\left(\mathcal{U}^{1}\right)_{f} \times-\alpha}\right) \otimes \mathcal{P}} \cong \Phi_{\left(1 \times g^{-1}\right)^{*} \mathcal{P}} \cong \Phi_{\mathcal{P}} \circ g^{*} .
$$

By putting all this together, we arrive at

$$
T_{\left.\mathcal{P}\right|_{\left(\mathcal{U}^{1}\right)_{f} \times-\alpha}} \circ f^{*} \circ \Phi_{\mathcal{P}} \cong T_{\left.\mathcal{P}\right|_{\left(\mathcal{U}^{1}\right)_{f} \times-\alpha}} \circ \Phi_{\mathcal{P}} \circ T_{\left.\mathcal{P}\right|_{\beta \times \mathcal{V}^{1}}} \cong \Phi_{\mathcal{P}} \circ g^{*} \circ T_{\left.\mathcal{P}\right|_{\beta \times \mathcal{V}^{1}}} .
$$


Therefore we have

$$
T_{\left.\mathcal{P}\right|_{\left(\mathcal{U}^{1}\right)_{f} \times-\alpha}} \circ f^{*} \circ \Phi_{\mathcal{P}} \cong \Phi_{\mathcal{P}} \circ T_{g^{*}\left(\left.\mathcal{P}\right|_{\beta \times \mathcal{V}^{1}}\right)} \circ g^{*} .
$$

Equation (6.5) shows that the presentation ${ }_{(L, \theta, \eta)} X_{f}$ is $\Phi_{\mathcal{P}}$-compatible (see Definition (5.6), and therefore the gerbe ${ }_{-\alpha} X_{\beta}$ is $\Phi_{\mathcal{P}}$-compatible, and so we can calculate the dual gerbe on a twisted version of $Y$. Therefore, by the lemma we have a dual gerbe, on the presentation $Y_{g}$ of $Y_{\alpha}$, given by $M=g^{*}\left(\left.\mathcal{P}\right|_{\beta \times \mathcal{V}^{1}}\right) \rightarrow \mathcal{V}^{1}$, along with some isomorphisms $T_{i j k}: M_{j k} \otimes g_{j k}^{*} M_{i j} \rightarrow M_{i k}$ and $\zeta_{i}: \mathcal{O} \rightarrow M_{i i}$ satisfying the diagrams of a gerbe presentation. According to the above discussion, the equivalence class of this gerbe on $Y_{\alpha}$ comes from the term in $E_{\infty}^{1,1}$ given by $[[M]]=\left[\left[g^{*}\left(\left.\mathcal{P}\right|_{\beta \times \mathcal{V}^{1}}\right)\right]\right]=\left[\left[\left.\mathcal{P}\right|_{\beta \times \mathcal{V}^{1}}\right]\right]$. This is the gerbe on $Y_{\alpha}$ which comes from $\beta \in H^{1}(B, \mathcal{X})$, and so we are done.

Remark 6.3. Clearly, we can also prove a more general statement by invoking Theorem 5.12. We leave the recording of this statement to the interested reader.

6.2. The conjecture of Donagi and Pantev. In this section we use a special case of the Corollary 6.2 and use it to reprove the main results in [19] as well as the following conjecture [19] of Donagi and Pantev, which was proven in [19] in many important special cases.

Conjecture 6.4. Let $X$ be a complex manifold elliptically fibered with at worst $I_{1}$ fibers over a normal analytic variety $B$ such that $H^{2}\left(B, \mathcal{O}^{\times}\right)=\{1\}$. Let $\alpha, \beta \in$ $\amalg_{a n}(X)$ be complementary elements (see [19] for the definition). Then there exists an equivalence

$$
D_{c}^{b}\left({ }_{\beta} X_{\alpha},-1\right) \cong D_{c}^{b}\left({ }_{\alpha} X_{\beta}, 1\right)
$$

of the bounded derived categories of sheaves of weights \pm 1 on ${ }_{\alpha} X_{\beta}$ and ${ }_{\beta} X_{\alpha}$ respectively.

Proof. Let $Y=X$ and let $\mathcal{X}$ be the sheaf of sections of $X$, considered as a sheaf of groups via a choice of a section $\sigma$. We have two flat morphisms, $\pi: X \rightarrow B$ and $\rho: X \rightarrow B$, giving $X$ two different structures of a reasonable torus fibration over $B$. We then get an isomorphism

$$
\amalg_{a n}(X) \cong H^{1}(B, \mathcal{X}) .
$$

Consider the rank one divisorial sheaf on $X \times_{B} X$ defined as

$$
\mathcal{P}=\mathcal{O}_{X \times_{B} X}\left(\Delta-\sigma \times_{B} X-X \times_{B} \sigma-\varpi^{*} N_{\sigma / X}\right),
$$

where $\varpi: X \times_{B} X \rightarrow B$ is the natural projection. It gives an equivalence of derived categories $\Phi: \mathrm{D}_{c}^{b}(X) \rightarrow \mathrm{D}_{c}^{b}(X)$ (see for example the paper of Bridgeland and Maciocia [10]). Furthermore, as explained in [19], $\mathcal{P}$ is a bi-extension. The fact that $\alpha$ and $\beta$ are complimentary (the triviality of the pairing $\left\langle S_{\alpha}(\beta), S_{\beta}(\alpha)\right\rangle$ from [19]) is equivalent to the vanishing of the images of $S_{\alpha}(\beta)$ and $S_{\beta}(\alpha)$ in $H^{3}\left(B, \mathcal{O}^{\times}\right)$ as explained in [19]. Now an application of Corollary 6.2 immediately produces an isomorphism as in (6.6) and hence proves Conjecture 6.4 of Donagi and Pantev [19.

Remark 6.5. In fact, since genus one fibrations with only nodes and cusps as singularities are reasonable complex torus fibrations, we have expanded the context in which their conjecture holds to this case. Of course, an even more general result for elliptic fibrations is given if we use Theorem 5.12 


\section{Conclusions AND SPECUlations}

In this section, we comment on some possible future generalizations of our main theorem. After this, we indicate a few theorems that we expect to be consequences or analogues of the results of this thesis. First of all, we expect to be able to dispense (see [16]) with the compactness and smoothness assumptions of our varieties, as well as to be able to extend our main result to the case where the fibrations are not flat, and perhaps the Poincaré sheaf is a more general object in the derived category of the fiber product. Certainly, we could replace our Serre functor with a suitable object in a singular situation. Compactness assumptions can be dispensed with by considering derived categories of compactly supported sheaves. The key ingredient needed for generalizations seems to be to find a diagram of isomorphisms of sheaves or complexes of sheaves which induce the diagram of natural equivalences (5.21). In many cases this should be possible using the constraints on the Poincaré object coming from the fact that it implements an isomorphism. Another strategy towards finding an object on the fiber product of the two gerbes is to use the technique of cohomological descent for gluing objects in the differential graded categories: Locally the object is just the Poincaré object, and the diagram of natural equivalences (5.21) becomes a descent data diagram in the differential graded category of sheaves on the fiber product.

Ideally, there would also be a presentation-free description of the twisting of derived equivalences. A scheme to do precisely this was suggested to us by D. Arinkin during a discussion of the original version of this paper. It is an extension of his duality for group stacks perspective, as found in the appendix to [19. We hope that future results generalizing those of our main theorem will find presentation-free descriptions.

In the search for applications of our main theorem to fibrations where the generic fiber has no group structure, a natural place to start is with $K 3$ fibrations. However, as we now explain, the most naive attempts seem to lead back to scenarios which are already understood. First, we start with two $K 3$ surfaces $W$ and $Z$ such that $Z$ is a fine moduli space for stable sheaves of Mukai vector $(r, H, s)$ on $W$. If $L$ is any line bundle on $W$, then the map

$$
T_{L}: \mathrm{D}_{c}^{b}(W) \rightarrow \mathrm{D}_{c}^{b}(W)
$$

acts on the Mukai vector by $T_{L}(r, H, s)=\left(r, r c_{1}(L)+H, s+\left(c_{1}(L), H\right)+\frac{1}{2} r c_{1}(L)^{2}\right)$. Since $c_{1}(L)$ is non-torsion, $r$ must then be zero in order for $T_{L}$ to preserve the moduli problem, and it can be used to build gerbes with the derived equivalence between $Z$ and $W$. Unfortunately, this together with the fact that $(r, H, s)^{2}=0$ implies that $H^{2}=0$. Now we can find a non-trivial line bundle $M$ such that $c_{1}(M)=H$. Then $M$ defines an elliptic fibration $W \rightarrow \mathbb{P}^{1}$. The sheaves on $W$ corresponding to points on $Z$ will all be supported on the fibers of this elliptic fibration. This argument seems to go through in the relative case (where $(r, H, s)$ now becomes a relative Mukai vector), and this shows that a $K 3$ fibration $W \rightarrow B$ admitting gerbes compatible with a derived equivalence (which are not pull-backs from $B$ ) becomes an elliptic fibration over a $\mathbb{P}^{1}$ fibration over $B$, and the derived equivalence respects this elliptic fibration structure. If it works, this reasoning will only be bi-rational, and refining it to an actual geometric statement may lead to new applications to $K 3$ fibrations. Also, note that if we replace our $K 3$ fibration with a, say, abelian surface fibration, then we could have $r$ be non-zero, provided that $L$ was $r$-torsion, 
and hence we expect a straightforward application of our main theorem in that case.

A perhaps more obvious application concerns the moduli spaces of vector bundles on curves. For concreteness, let $C$ be a genus 2 hyperelliptic curve. Then from the work of Desale and Ramanan [17] we recall that the moduli space of (isomorphism classes of stable) vector bundles of rank 2 and the fixed odd determinant of odd degree is isomorphic to the intersection $X=Q_{1} \cap Q_{2}$ of two quadric four-folds in $\mathbb{P}^{5}$. Bondal and Orlov, in [7, give an explanation in terms of derived categories. They produce a vector bundle $V$ on $C \times X$ that implements a full and faithful embedding $\mathrm{D}_{c}^{b}(C) \rightarrow \mathrm{D}_{c}^{b}(X)$. Finally they prove that this morphism induces a semiorthogonal decomposition:

$$
\mathrm{D}_{c}^{b}(X)=\left\langle\mathcal{O}_{X}(-1), \mathcal{O}_{X}, \mathrm{D}_{c}^{b}(C)\right\rangle
$$

Now let $\pi: C \rightarrow B$, factorizing as $C \rightarrow P \rightarrow B$, be a smooth compact family of non-singular hyperelliptic curves over a projective bundle $P$ given by 6 sections of $P$ over $B$. Let $\mathfrak{C}$ be a gerbe on $C$, presentable by a collection of line bundles $M_{i j}$ on $\pi^{-1}\left(U_{i j}\right)$ which are 2-torsion on each hyperelliptic fiber. Conjugating these line bundles by the Fourier-Mukai transform (using a left adjoint) gives locally automorphisms $f_{i j}$ of a natural family of quadrics $X$ inside a $\mathbb{P}^{5}$ bundle over $B$. By gluing we obtain a new family of quadrics $\tilde{X} \rightarrow B$. A simple application of the main theorem should give a full and faithful embedding $\mathrm{D}_{c}^{b}(\mathfrak{C},-1) \rightarrow \mathrm{D}_{c}^{b}(\widetilde{X})$. The line bundle $\mathcal{O}_{X}(-1)$ will induce a line bundle $\mathcal{O}_{\widetilde{X}}(-1)$, and the embedding will induce a semiorthogonal decomposition:

$$
\mathrm{D}_{c}^{b}(\widetilde{X})=\left\langle\mathcal{O}_{\widetilde{X}}(-1), \mathcal{O}_{\widetilde{X}}, \mathrm{D}_{c}^{b}(\mathfrak{C},-1)\right\rangle
$$

7.1. Relation to mirror symmetry and geometric langlands. Finally, let us explain a simple example of the type of idea in homological mirror symmetry that our main theorem might lead one to conjecture. We do not give a complete set of references here, but for what we are going to discuss, 1] and the references therein are relevant. We would like to propose an extension of the usual ways that gerbes are thought to enter the homological mirror symmetry story [22, [19. However, our analysis applies to a different type of gerbe. Let $B_{1}=F_{1}=B_{2}=F_{2}=S^{1}$. Let $E_{1}=B_{1} \times F_{1}$ and $E_{2}=B_{2} \times F_{2}$ be elliptic curves. We assume that dualizing $F_{i}$ gives homological mirror symmetry identifications $\mathrm{D}_{c}^{b}\left(E_{i}\right) \cong \operatorname{DF} u k\left(S_{i}\right)$, which induce isomorphisms if we base change everything via the inclusion of open sets in the base $B_{i}$. Here $S_{i}=B_{i} \times F_{i}^{\vee}$ are symplectic tori of real dimension 2. Let $X=E_{1} \times E_{2}$ and $Y=S_{1} \times S_{2}$. We also assume we can take the "product" of equivalences, and so we also have the equivalence $\Phi: \operatorname{DF} u k(Y) \rightarrow \mathrm{D}_{c}^{b}(X)$, which induces isomorphisms if we base change everything via the inclusion of open sets in $B_{1}$ (we will not care about the analogous property for $B_{2}$ ). Here $\mathrm{D} F u k(Y)$ should include the coisotropic branes of Kapustin and Orlov; see [24. Consider the projections

$$
\pi: X \rightarrow B_{1}, \quad \rho: Y \rightarrow B_{1} \quad \text { and } \quad \mu: X \rightarrow E_{2} .
$$

Embed $E_{2}$ into a projective space; this gives us a line bundle $\mathcal{O}(1)$ on $E_{2}$. Fix a class $\left\{n_{i j}\right\} \in \check{Z}^{1}\left(B_{1}, \mathbb{Z}\right)$ for some small open cover $\left\{U_{i}\right\}$ of $B_{1}$. Consider an $\mathcal{O}^{\times}$gerbe $\mathfrak{X}$ on $X$ presented by the collection of line bundles $\left\{\mu^{*} \mathcal{O}\left(n_{i j}\right) \rightarrow \pi^{-1}\left(U_{i j}\right)\right\}$. Now conjugating the tensorization by $\mu^{*} \mathcal{O}\left(n_{i j}\right)$ by the (appropriately restricted) transformations $\Phi_{U_{i j}}$ yields automorphisms $g^{n_{i j}}$ of $\rho^{-1}\left(U_{i j}\right)=U_{i j} \times F_{1}^{\vee} \times S_{2}$. These are symplectomorphisms of $\rho^{-1}\left(U_{i j}\right)$ which are constant on the $U_{i j} \times F_{1}^{\vee}$ factor and 
which are given by $n_{i j}$ compositions of a Dehn twist $g$ on the factor $S_{2}$. Using them, we can produce a new symplectic 4-manifold $\tilde{Y}$.

Then, by analogy with the main theorem, we can conjecture that

$$
\mathrm{D}_{c}^{b}(\mathfrak{X},-1) \cong \operatorname{DFuk}(\tilde{Y}) .
$$

Notice that the automorphisms destroy the second $S^{1}$ fibration structure, but do nothing to the first one. By contrast, if the $\mathcal{O}(1)$ was replaced by a line bundle flat along the $F_{2}$ direction, we would expect to keep both $S^{1}$ fibration structures on the symplectic side, fixing the first one and turning the second into a non-trivial principal $S^{1}$ bundle.

Finally, we would like to mention a recent paper of Donagi and Pantev on the duality between the Hitchin integrable systems associated to a simple complex Lie group and its Langlands dual. There, they prove a derived equivalence between the bounded, compactly supported, coherent derived categories of the commutative group stacks ${ }^{L} \mathcal{H}$ iggs and $\mathcal{H}$ iggs, over a space $B-\Delta$. Here $\Delta$ is a discriminant locus over which the Hitchin fibration has singular fibers. They remark there that the results in the thesis can be used in their proof, and further should be useful to extend the duality over all of $B$. For a more complete explanation, we refer to their paper [20].

\section{ACKNOWLEDGMENTS}

The author would like to thank Tony Pantev for supervising his thesis and for his indispensable help and reading of this manuscript. Thanks also to Jonathan Block, Ron Donagi, Jim Stasheff, and Dima Arinkin, and many others, for interesting conversations and comments on the original draft of the paper. Thanks to the referee for finding several typos.

\section{REFERENCES}

[1] M. Aldi and E. Zaslow, Seidel's mirror map for abelian varieties, 2005, arXiv:math.SG/ 0512229 .

[2] B. Andreas and D. Hernández Ruipérez, Fourier Mukai transforms and applications to string theory, RACSAM Rev. R. Acad. Cienc. Exactas Fís. Nat. Ser. A Mat. 99 (2005), no. 1, 29-77. MR2174259

[3] D. Arinkin, Fourier transform for quantized completely integrable systems, Ph.D. thesis, Harvard University, 2002

[4] O. Ben-Bassat, J. Block, and T. Pantev, Non-commutative tori and Fourier-Mukai duality, 2005, math.AG/0509161, to appear in Compositio Mathematica.

[5] J. Block, Duality and equivalence of module categories in noncommutative geometry I, 2005, math.QA/0509284.

[6] _ Duality and equivalence of module categories in noncommutative geometry II: Mukai duality for holomorphic noncommutative tori, 2006, math.QA/0604296.

[7] A. Bondal and D. Orlov, Semiorthogonal decomposition for algebraic varieties, 1995, alggeom/9506012.

[8] T. Bridgeland, Equivalences of triangulated categories and Fourier-Mukai transforms, Bull. London Math. Soc. 31 (1999), no. 1, 25-34. MR1651025 (99k:18014)

[9] T. Bridgeland, A. King, and M. Reid, The McKay correspondence as an equivalence of derived categories, J. Amer. Math. Soc. 14 (2001), no. 3, 535-554 (electronic). MR1824990 (2002f:14023)

[10] T. Bridgeland and A. Maciocia, Fourier-Mukai transforms for K3 and elliptic fibrations, J. Algebraic Geom. 11 (2002), no. 4, 629-657. MR.1910263(2004e:14019) 
[11] V. Brinzanescu and R. Moraru, Twisted Fourier-Mukai transforms and bundles on nonKahler elliptic surfaces, Math. Res. Lett. 13 (2006), no. 4, 501-514. MR2250486 (2007e:32018)

[12] U. Bunke, P. Rumpf, and T. Schick, The topology of T-duality for Tn-bundles, 2005, math.GT/0501487.

[13] I. Burban and B. Kreussler, On a relative Fourier-Mukai transform on genus one fibrations, 2004, math.AG/0410349.

[14] A. Căldăraru, Derived categories of twisted sheaves on calabi-yau manifolds., Ph.D. thesis, Cornell University, 2000.

[15] _ Derived categories of twisted sheaves on elliptic threefolds, J. Reine Angew. Math. 544 (2002), 161-179. MR.1887894 (2003a:14022)

[16] D. Hernández Ruipérez, A.C. López Martín, and F.S. de Salas, Fourier-Mukai transforms for Gorenstein schemes, Adv. Math. 211 2007, 594-620. MR2323539 (2008e:14019)

[17] U. V. Desale and S. Ramanan, Classification of vector bundles of rank 2 on hyperelliptic curves, Invent. Math. 38 (1976/77), no. 2, 161-185. MR0429897|(55:2906)

[18] Igor Dolgachev and Mark Gross, Elliptic threefolds. I. Ogg-Shafarevich theory, J. Algebraic Geom. 3 (1994), no. 1, 39-80. MR.1242006 (95d:14037)

[19] R. Donagi and T. Pantev, Torus fibrations, gerbes, and duality, Mem. Amer. Math. Soc. 193 (2008), vi + 90 pp. MR2399730

[20] Langlands duality for Hitchin systems, 2006, arXiv:math.AG/0604617.

[21] P. Gabriel, Des catégories abéliennes, Bull. Soc. Math. France 90 (1962), 323-448. MR0232821 (38:1144)

[22] Nigel Hitchin, Lectures on special Lagrangian submanifolds, Winter School on Mirror Symmetry, Vector Bundles and Lagrangian Submanifolds (Cambridge, MA, 1999), AMS/IP Stud. Adv. Math., vol. 23, Amer. Math. Soc., Providence, RI, 2001, pp. 151-182. MR 1876068 (2003f:53086)

[23] D. Huybrechts, Fourier-Mukai transforms in algebraic geometry, IJM Paris, Oxford University Press, 2006. MR2244106 (2007f:14013)

[24] Anton Kapustin and Dmitri Orlov, Remarks on A-branes, mirror symmetry, and the Fukaya category, J. Geom. Phys. 48 (2003), no. 1, 84-99. MR2006226 (2004f:14053)

[25] M. Kashiwara and P. Schapira, Sheaves on Manifolds, Grundlehren der Mathematischen Wissenschaften [Fundamental Principles of Mathematical Sciences], vol. 292, Springer-Verlag, Berlin, 1990, With a chapter in French by Christian Houzel. MR.1074006 (92a:58132)

[26] M. Lieblich, Moduli of Twisted Sheaves, 2004, arXiv:math.AG/0411337.

[27] V. Mathai and J. Rosenberg, T-duality for torus bundles with $H$-fluxes via noncommutative topology, Comm. Math. Phys. 253 (2005), no. 3, 705-721. MR2116734

[28] _ T-duality for torus bundles with H-fluxes via noncommutative topology, II: the highdimensional case and the T-duality group, 2005, hep-th/0508084.

[29] S. Mukai, Duality between $D(X)$ and $D(\hat{X})$ with its application to Picard sheaves, Nagoya Math. J. 81 (1981), 153-175. MR.82f:14036

[30] A. Polishchuk, Abelian varieties, theta functions and the Fourier transform, Cambridge Tracts in Mathematics, vol. 153, Cambridge University Press, Cambridge, 2003. MR 1987784

[31] J. Sawon, Twisted Fourier-Mukai transforms for holomorphic symplectic fourfolds, 2005, arXiv:math.AG/0509222.

[32] Y. Toda, Deformations and Fourier-Mukai transforms, 2005, math.AG/0502571.

Einstein Institute of Mathematics, Edmond J. Safra Campus, Givat Ram, The Hebrew University of Jerusalem, Jerusalem, 91904, IsRael

E-mail address: oren.benbassat@gmail.com 